\title{
Philosophiques
}

\section{Pouvoir de la culture et culture du pouvoir}

\section{André Paradis}

Volume 14, numéro 1, printemps 1987

URI : https://id.erudit.org/iderudit/027003ar

DOI : https://doi.org/10.7202/027003ar

Aller au sommaire du numéro

\section{Éditeur(s)}

Société de philosophie du Québec

ISSN

0316-2923 (imprimé)

1492-1391 (numérique)

Découvrir la revue

Citer cet article

Paradis, A. (1987). Pouvoir de la culture et culture du pouvoir. Philosophiques, 14(1), 57-119. https://doi.org/10.7202/027003ar

\section{Résumé de l'article}

Passant par les voies non pavées de l'interdisciplinarité, on entend ici montrer, au moyen d'une réflexion sur la triple articulation du corps, du signe et du pouvoir, en quoi l'alternative classique et binaire du matérialisme et de l'idéalisme peut être dépassée. Par l'approche utilisée, on ambitionne de contribuer conjointement à la théorie de la pensée et des représentations socio-culturelles et à une critique du concept de pouvoir revu à la lumière de la socio-psychanalyse. Les notions d'émancipation et de création, parallèlement à celles de soumission et d'aliénation, sont reprises dans la perspective des conditions socio-culturelles de l'accès à la parole et à l'identité aussi bien personnelle que groupale et collective.
Ce document est protégé par la loi sur le droit d'auteur. L'utilisation des services d'Érudit (y compris la reproduction) est assujettie à sa politique d'utilisation que vous pouvez consulter en ligne.

https://apropos.erudit.org/fr/usagers/politique-dutilisation/ 
PHILOSOPHIQUES, Vol. XIV, Numéro 1, Printemps 1987

\title{
ARTICLES \\ POUVOIR DE LA CULTURE ET CULTURE DU POUVOIR
}

\author{
par André Paradis
}

\begin{abstract}
RÉSUMÉ. Passant par les voies non pavées de l'interdisciplinarité, on entend ici montrer, au moyen d'une réflexion sur la triple articulation du corps, du signe et du pouvoir, en quoi l'alternative classique et binaire du matérialisme et de l'idéalisme peut être dépassée. Par l'approche utilisée, on ambitionne de contribuer conjointement à la théorie de la pensée et des représentations socio-culturelles et à une critique du concept de pouvoir revu à la lumière de la sociopsychanalyse. Les notions d'émancipation et de création, parallèlement à celles de soumission et d'aliénation, sont reprises dans la perspective des conditions socio-culturelles de l'accès à la parole et à l'identité aussi bien personnelle que groupale et collective.
\end{abstract}

ABSTRACT. Borrowing the unpaved ways of interdisciplinarity we will here try to show, relying upon reflections on the relations between the body, signs and power, how the traditional and binary opposition of materialism and idealism can be overcome. It is hoped that the approach will jointly be a contribution to a theory of thought and of socio-cultural representations and a critique of the concept of power, restated with the assistance of socio-psychoanalysis. The notions of liberation and of creation, with those of submission and alienation, are here recaptured in connection with the socio-cultural conditions of access to language (parole) and to individual as well as group and collective identity.

\section{DE L'ÂME ET DU CORPS OU DE NOTRE PASSION POUR LES ANTINOMIES}

Toutes les sciences de la culture ont leurs présupposés philosophiques et toutes les philosophies leurs postulats anthropologiques. C'est ainsi que depuis Descartes, de façon tout à 
fait explicite, mais, pourrions-nous opiner tout autant, depuis les débuts mêmes de la pensée et de la culture ococidentales, nous avons été amenés à choisir plus ou moins obscurément entre une métaphysique de l'Esprit et une physique du corps mécanique, entre l'homme de bien ou de génie qui voue son existence au triomphe de la Raison, de la libre pensée et de la Vertu et l'homme passionnel, soumis à la loi aveugle de la Nature et qui parcoure ainsi, sans grand mérite, la voie déjà toute tracée de la Nécessité. D'un côté donc un chant exaltant l'accès ou la participation à la transcendance, de l'autre un refrain commémorant la répétitivité du Destin. Ainsi placés dans la proximité antipathique mais bien réglée d'un salubre voisinage, religion et science profane de la nature aussi bien qu'âme et corps, liberté et passion, conception et sensation se trouvaient voués à un dialogue tout aussi forcé qu'inopérant, stérile et impossible. Le partage du monde étant bel et bien ainsi convenu, ne point respecter les bornes et les frontières de chaque univers et par là même leur indissociable corrélativité dans l'exclusion eut assurément mené à franchir le tabou suprême: proclamer sans métaphore que la pensée fut corporelle et que le corps fut intelligence, sans qu'il y ait ici de privilège accordé ou à l'un ou à l'autre, c'eut assurément été non seulement retomber dans la confusion d'un certain panthéisme vite mis au rancart de la tradition intellectuelle ou fréquenter les parvis douteux de l'atomisme et de l'épicurisme, mais rendre tout autant inintelligibles les critères éminemment spéculatifs permettant de bien discriminer qui est religieux et qui ne l'est pas, qui est matérialiste et empiriste et qui est rationaliste et spiritualiste, qui est croyant et qui est fataliste, qui convient de l'autonomie de la volonté et qui nous voue à la passivité, à l'irresponsabilité ou à la servilité de nos déterminismes corporels. C'eut même peut-être été remettre en cause, j'en conviens, la crédibilité même de la science pour autant que la science ne pouvait elle-même, dans un tel contexte, que signer à sa manière, le triomphe de la pensée sur la matière, de l'âme rationnelle sur l'empiricité nä̈ve et tâtonnante des sens, de Dieu lui-même sur le monde, pour autant que par notre intelligence et donc par notre science nous serions également à son image. Ainsi, à l'époque même de la grande Renaissance et des premiers balbutiements du mécanisme ouvertement déclaré, la science, de par la partie noble de son projet, à savoir l'esprit, pouvait-elle s'accommoder de 
demeurer encore foncièrement religieuse tout en maintenant, de par la matérialité de son objet, son allégeance aux nouveaux canons de l'empirisme naissant. Tout comme il eut suffit ici à un Galilée récalcitrant et mauvais causeur d'avouer que tout dans le monde physique semble bien se passer comme si, selon les apparences, mais point davantage pour ne point encourir les foudres de Rome, de même pour que la religion cédât de son côté aux avances de la science, il eut sans doute suffi, dans ce même contexte, que l'esprit se prit lui-même comme objet de science, ce qui, par définition convenue, était toutefois manifestement impossible, l'esprit étant d'emblée pour lui-même, et par essence, transparent et immédiatement donné : tel devait être du moins le sens de l'une des implications du cogito cartésien en vertu duquel la conscience ne pouvant être que la totalité du sujet qui nous rend ainsi maîtres de nos propres clartés, le corps du sujet ne pouvait dès lors être lui-même, à proprement parler, moins encore que la médiation, que le médium de notre subjectivité pensante, en d'autres termes ce par quoi nous sommes rendus présents au monde plus que ce en quoi nous sommes cette présence (ou cette absence) même au monde. En cette façon encore toute péripatéticienne de voir les choses, les mots corps et esprit, signifiant encore deux royaumes bien distincts, ne pouvaient signifier encore que deux substances. On comprend donc que, pour Descartes, déjà gagné à ces deux royaumes et ne voulant avec raison en sacrifier aucun, la glande pinéale demeura le lieu symbolique et inexpugnable d'un paradoxe non résolu et qu'à Elizabeth, dont la candeur naïve valait bien plusieurs têtes de philosophes, Descartes se vit dans l'obligation de répondre que l'union de l'âme et du corps devait bien constituer quelque chose comme une troisième substance ${ }^{1}$. On doit croire que cette façon de maintenir sa croyance légitime en nos deux substances en en alléguant une troisième concomittante n'était pas qu'un simple artifice verbal; mieux valait en tout état de cause démultiplier les substances au besoin et garder ainsi malgré tout un pied bien campé dans les plates-bandes

1. Cf. René Descartes, lettres à Élisabeth du 21 mai et du 28 juin 1643, in Descartes, Euvres philosophiques, t. III (1643-1650), édition de F. Alquié, Garnier, 1973, pp. 19, 44 (note 2), 47 et 48 ; cf. aussi l'Abrégé des Médiations et le commentaire de Germaine Lot, in Descartes, éd. Seghers, coll. savants du monde entier, $\mathrm{n}^{\circ} 30$, Paris, 1966 , Pp. 84-85. 
de l'empirisme et de la sensorialité que de risquer de passer pour un obscurantiste en alléguant tout simplement l'argument du mystère ontologique de l'union consubstantielle du corps et de l'âme; et mieux valait tout autant garder l'autre pied bien campé dans le camp de la rationalité théologique pour autant que la Raison mathématique, maintenue ainsi dans la transcendance indiscutable de sa propre nature, pouvait elle-même s'avérer garante de son propre régime d'idées claires, fût-ce sous le regard encore bienveillant d'un Dieu bon qui ne saurait assurément se laisser aller à l'infatuation et aux velléités capricieuses d'un mauvais génie. Ainsi la philosophie, fut-ce au prix d'une contorsion qui pourra nous paraître un peu abusive aujourd'hui, pouvait-elle demeurer un lieu de répit, de délai et de réflexion relativement autonome et distinct de la foi sans que l'on ne soit contraint pour autant de renier les fondements de la métaphysique et de la théodicée classiques. L'esprit demeurait bien l'esprit et la matière la matière.

Cette double tentation du matérialisme et de l'idéalisme s'était à vrai dire, bien avant Descartes, toujours avérée dilemmatique puisque de la réalité du corps aussi bien que de celle de la pensée nous ne saurions rien renier. On pourrait même se risquer à penser que c'est l'énigme même de notre double nature et notre insatiable passion pour réconcilier et réunir en une identité ce qui se donne à voir en nous sous le mode d'une irréductible antithèse qui devaient, dès l'origine, s'avérer être le moteur de toute pensée réflexive et de toute entreprise d'humanisation et de civilisation. Comment, en effet, réunir quelque part, dans une proximité aussi étroite que hiérarchisée, sans pour autant les confondre, des termes qui ne semblaient pouvoir signifier, en regard de l'expérience la plus immédiate, que sous le signe et que sous la conjonction d'une profonde division? Comment en arriver, en effet, à domestiquer la Discorde sans lui opposer conjointement et dialectiquement la Concorde, les forces dissolvantes de la Haine sans leur opposer le pouvoir intégrateur d'Eros, la matière informe et chaotique sans la circonvenir et la façonner par la Forme, les atomes éternellement mobiles en leur errance aléatoire sans les soumettre à la contrepartie de l'Intelligence ordonnatrice (Nous), le Multiple et le Mobile sans les réduire à n'être que l'Apparence de la rotondité de l'Un, le devenir capricieux des 
accidents sans les subordonner conjointement à la perdurance de la Substance? Comment contrevenir à la discontinuité précaire de nos sensations, par nature fugitives et éphémères et qui, à la limite, nous contraindraient à laisser notre jugement en suspens, sans mobiliser contre elles les forces de résistance rassurantes de l'illumination divine, des formes a priori de notre entendement ou cette finalité obscure, mais non moins soi-disant omniprésente, de l'Évolution créatrice qui prélude à l'explosion de tout élan vital? Comment, en somme, rester maître du Hasard et de la dispersion sans postuler quelque part le pouvoir et l'efficace d'une Loi intelligible et ordonnatrice de toutes choses?

\section{PLATON ET LE DILEMME DE L'ESTHÉTIQUE}

ET DE L'IDÉE : OU DE LA DIFFICULTÉ ET DE LA TENTATION DE PENSER UN ENTRE-DEUX

À ce chapitre, Platon, qui est à la fois le plus détesté et le plus aimé de tous les philosophes selon que l'on s'en tient strictement à la lettre ou que l'on croit en entrevoir l'esprit, est bel et bien demeuré le mâtre et le prototype de la culture philosophique occidentale bien qu'il soit une tradition exégétique qui me semble avoir vite malmené la concrétude de son intelligence en nous le livrant sous les auspices d'un mysticisme ou d'un rationalisme métaphysique peu rassurants. Car déjà chez lui, on peut saisir toute l'amplitude du dilemme philosophique qui nous le rend d'une part familier avec la tentation d'un idéalisme radical sans que nous ne puissions pour autant jamais conclure, d'autre part, qu'il soit étranger ou insensible à la tentation du matérialisme ou du sensualisme, ne serait-ce que parce qu'il s'en défend trop mais surtout parce qu'il n'est jamais de son dessein d'entreprendre d'en faire l'économie pour s'adonner à une contemplation dilettante et facile de l'Idée. Peut-être Platon aurait-il été d'ailleurs gravement offusqué et peut-être même aurait-il abondamment ironisé à juste titre sur notre penchant à vouloir le réduire à la sollicitation d'une nomenclature binaire, fut-ce pour mieux le porter aux nues ou pour mieux l'invectiver. En d'autres termes, Platon n'était peutêtre au fond ni idéaliste ni a fortiori matérialiste si tant est que l'on est en droit de penser que le corps, véritable voie labyrintique d'une réminiscence de l'Idée et s'avérant être en quelque sorte une occasion nécessaire pour (que naisse) l'Esprit plutôt que de lui 
faire unilatéralement obstacle, devenait alors, à ses yeux, partie prenante de toute entreprise de libération ou d'émancipation. Comment, en effet, accéder à la connaissance et à la science du politique qui est l'art de gouverner les hommes et de gérer justement l'avoir en regard de l'unité jalousement poursuivie de la Cité, comment accéder à la connaissance de la nature et des lois qui régissent son unité, comment accéder à la connaissance même des vérités théorématiques éternelles sans qu'entre l'Esprit et le Monde ne s'interposât à titre, disons encore de médium, la sensorialité aussi bien que la sensualité mathématique et esthétique de la figure et de l'image? N'est-ce point d'abord par le biais des beaux corps que pouvait germer le désir de la Beauté ellemême? N'est-ce point au cœur même de l'expérience particulière que pouvait fleurir le désir d'une compréhension plus universelle des choses tout comme, déjà chez Pythagore, n'est-ce point par le biais et le détour de la figure bien matérielle que le Nombre pouvait se révéler être l'essence même du Monde?

Rien, assurément, j'en conviens, ne lève encore ici la suspicion que l'on peut entretenir à l'endroit de Platon d'avoir succombé à un dualisme d'autant plus irréductible que le corps fut justement pour quelque chose dans le travail dascension dialectique conduisant jusqu'à l'embrassement de l'Idée. Rien ne peut nous prémunir sérieusement de le taxer de puriste, de platonique ou d'idéaliste. Et pourtant on peut aussi penser que ce dualisme toujours irrémédiablement maintenu, bien que peu à peu scotomisé, que cette tentative précaire, tragique et peut-être désespérée de réunir ce qui avait d'abord été pensé et vécu comme divisé n'était peut-être encore qu'une façon de dire, qu'une façon métaphorique de donner à penser qui, pour se prendre au sérieux, n'en arrivait pas pour autant à se clore et à se croire. Car, au fond, Platon, s'en prenant à son corps défendant contre les abus de la rhétorique des sophistes, aussi bien que contre les simulacres des artistes et des poètes, se doutait bien déjà que la pensée put être aussi infiniment passionnelle et donc corporelle, que le corps, par conséquent, put lui aussi se faire pensée, en sorte que la frontière substantielle, pour ne pas dire essentielle, qui avait d'abord été érigée entre l'Esprit et la Matière, put aussi s'avérer n'être rien d'autre en réalité qu'un banal petit poste de douaniers aux mours un peu laxistes. Sans doute Platon aurait-il été plus réticent à 
admettre que la pensée vertueuse, que la philosophie elle-même, récalcitrante aux subterfuges de la rhétorique et de l'esthétique put, elle aussi, être infiniment corporelle. Car c'eut été non seulement admettre l'excellence de l'art et de la rhétorique comme œuvre médiatrice du signe et comme principauté incontournable du signifiant, là même où l'Idée voudrait être poursuivie et contemplée pour elle-même et en elle-même, mais remettre aussi sérieusement en question l'échelle ontologique des valeurs en vertu de laquelle l'inférieur ne peut se dire définitivement qu'à propos du supérieur, l'image qu'à propos de l'Idée immuable, qu'elle ne peut au mieux que préfigurer tout en la masquant, que laisser à deviner tout en la dissimulant à travers le voile opaque et éphémère de ses espaces et de ses formes.

Mais, là encore, on ne saurait être trop prudent et peut-être vaut-il mieux se garder de précipiter l'interprétation. Car, pour Platon, la philosophie était bel et bien une passion amoureuse pour la sagesse, une quête et une chasse amoureuse de vérité. Et peut-être ce parti-pris passionné (plutôt que passionnel...), qui n'a guère à voir avec l'indifférence ou la neutralité que l'on consent habituellement avec respect ou avec mépris aux intellectuels, trahissait-il bien que toute pensée intense, créatrice, éprise de révélation philosophique, poétique et même mathématique, est infiniment corporelle elle aussi à moins que, se rangeant du côté du Tout ou du côté du Rien, dans l'idée que la binarité est en soi une vertu ou une obligation, Platon ait préféré croire que la Raison a ses passions que le corps ne connaît pas. Laissons-nous plutôt aller à croire, ne fut-ce qu'un instant, que si le prisonnier de Platon, qui a rompu ses chaînes, se trace en tâtonnant la voie qui le mène jusqu'à l'orée de la caverne et jusqu'à la fulgurance de l'Idée solaire, ce n'est point pour s'enfuir dans un au-delà enchanteur, dans une délectation mystique et suprême, mais pour mieux réintégrer ses propres quartiers et pour mieux infléchir les ombres, les figures et les images en faveur de son projet trop humain de vérité. Ce ne saurait être en tout cas pour répudier les ombres ou pour les condamner, puisque de cette Idée Solaire, dont il aurait peut-être été le témoin, Platon, aveuglé dit-on, n'aurait pu lui-même en soutenir la vision face à face. Peut-être n'y aurait-t-il cueilli, en cette Idée du Bien, en cet intangible Absolu qui éclaire toute réalité 
visible, en cette inéluctable transcendance de la Réalité et de l'Ensoi, que le rappel de sa propre finitude oubliée, que l'éveil de sa propre conscience au champ de l'illusion comme inscrit au coeur même de la condition humaine, que le rappel donc de sa vocation à tendre vers un au-delà des apparences là où, en proie au jeu des ombres et en butte à un rêve déchirant, toujours renaissant et toujours évanouissant d'Idéalité, il ne s'était jusqu'alors perçu luimême que sous le signe d'une fatale et intolérable condamnation. Ainsi le prisonnier platonicien, à peine émancipé de ses chaînes, fait-il retour dans sa caverne qui demeure bel et bien pour lui le seul lieu habitable, si humide soit-il, contre l'intempérie. Et sa seule certitude, qui pourrait dès lors le rendre suspect et étranger aux yeux des siens, ne serait donc point de savoir la vanité des signes puisque de signes ou d'ombres nous ne saurions manifestement nous passer, mais leur bienveillante ou malveillante ambiguité. Ainsi ce qu'après Socrate nous dénommons le doute philosophique ne serait-il que l'acceptation vigilante des conditions et des limites de notre humanité et le maintien du désir, toujours insatisfait, au cœur de ce que nous ne cessons de vouloir lui offrir en pâture et à titre de signes en sorte que le Réel, par-delà ce qu'en dit la doxa, qui est le lieu de l'intérêt et du pouvoir, puisse aussi avoir quelque chance de nous être signifié. Car si des signes nous avons la passion idolâtre nous en avons aussi le génie mais ce génie ne va assurément jamais sans que l'on n’y risque aussi sa propre méprise. Le dialogue est toujours une entreprise passionnante mais toujours aussi, admettons-le, une entreprise périlleuse.

PENSER LA PENSÉE EN TERMES DE DÉSIR ET

D'HISTOIRE PLUTOOT QU'EN TERMES DE SUBSTANCE ?

On comprend dès lors que si le philosophe entretient toujours une saine abstention à partager ce qui se donne très tôt ou trop tôt sous le signe d'une parfaite évidence, que ce soit celle de la tradition ou celle d'une doxa trop avide de rallier les crédulités dans l'absolu d'un consensus apaisant et rassurant ${ }^{2}$, il puisse en cela même être accusé, ainsi que Socrate, d'être un sans dieu. Car

2. Entendons ici les cultes religieux, politiques, philosophiques officiels. 
rien ne saurait désormais le soustraire à la conscience de la nécessité et de la précarité des signes et des images qui l'entraînent sur la voie de sa propre délivrance. De la réalité nous n'avons en effet de connaissance que celle qui se soutient des mots et se médiatise dans l'ordre du discours et du discours le sujet ne saurait lui-même se soustraire en tant qu'il est celui-là même qui, du creux de son propre désir et de sa propre parole, affirme et promulgue de façon prospective et risquée ce qu'il en est de la réalité du monde. Et c'est peut-être ici même que divergent les significations du doute cartésien et du doute socratique. Car si le doute de Descartes est infiniment épris de vérité apodictique, celui de Socrate, peutêtre plus païen, mais davantage religieux, est infiniment épris de foi et de fidélité dans la soutenance de notre condition qui en est une d'opacité pour nous-mêmes. Sans doute est-ce là la raison pour laquelle la philosophie ne se dit d'abord socratiquement que du travail de se connaître soi-même. La transcendance ne se dirait plus ici que de la recherche du sens et de l'intelligence de notre immanence comme notre âme ne se dirait plus ici que de la recherche du sens et de l'intelligence même de notre corps. Et notre corps ne se dirait plus lui-même ici que de son animation toute humaine, c'est-à-dire du sens et de l'intelligence qu'il peut ou ne peut arriver à faire pour lui-même dans son rapport au monde. Aussi ne saurait-on dès lors être ni idéaliste ni matérialiste, ni favorable à quelque troisième substance, la seule interrogation qui nous agrée étant sans doute de chercher à savoir comment le corps, à travers les signes, peut en arriver à se faire conscience et se rendre ainsi présent à lui-même et comment la conscience peut elle-même prendre corps en sorte que la subjectivité puisse se dire indistinctement et du corps et de l'âme, ces mots, ces signes, corps et âme, n'étant plus eux-mêmes qu'une façon de penser, selon un ordre syntagmatique, ce qui semble bien échapper au tamis et à l'ordre discret des mots mais en y laissant sa trace. Mais était-ce bien là vraiment la pensée de Platon ou ne serait-ce pas plutôt celle qu'à travers notre interprétation des signes platoniciens, et selon notre propre désir, nous lui prêterions nous-mêmes?

L'idée donc toute platonicienne, à mon sens, qui veut que tout parcours de vérité ne peut résulter que d'un travail laborieux de réminiscence, que ce travail est assurément périlleux puisqu'il ne peut se produire lui-même que sur le terrain mouvant et fracturé 
d'une doxa qui s'effrite et que par une sorte d'arrachement dialectique de l'étreinte du pouvoir adhésif des simulacres, que cet arrachement n'est point fuite mais plutôt réconciliation avec cela même que nous avons d'abord mais trop tôt aveuglément embrassé, selon l'ordre de la Nécessité et du Destin, qu'en cela même tout signe et tout simulacre portent bien en eux toute l'ambiguité esthétique d'une opacité ancienne aussi bien que d'une promesse à venir, cette idée, toute platonicienne ${ }^{3}$ dis-je bien, initiatrice de toute entreprise philosophique, aurait assurément dû tout au moins nous rendre sensibles à deux choses : d'une part que la conscience n'est point substance mais plutôt histoire et devenir ; d'autre part que toute subjectivité n'est point transparence et immédiateté pour soi-même, mais plutôt et d'abord érrangeté et écart à soi-même, absence à ce qui pourrait être dit, comme toute parole n'est point d'abord témoignage mais plutôt et d'abord aventure et errance, arrêts et résidences dans les excursus de la métaphore. Qu'en conséquence toute conscience, en proie à la puissance du signe et à l'émotion qui s'y investit, est donc fondamentalement corporelle en ce qu'elle est une ouvre du désir, comme tout désir humain ne peut se dire lui-même que des signes et des simulacres à travers lesquels il cherche à se signifier pour mieux se rendre présent à lui-même, ou pour se perdre davantage, pour mieux se posséder ou pour mieux se renoncer, pour mieux se partager dans les voies énigmatiques du dialogue ou pour mieux s'oublier dans l'écho vide d'un pur flatus vocis, le signifiant étant toujours libérateur ou geôlier de ce qui de nous-mêmes pourrait nous être signifié.

3. Ainsi serais-je tenté, en cette lecture tout improvisée de Platon, d'établir les équations suivantes : L'Idée $=$ L'En-soi $=$ La Réalité $=$ ce qui en tant même que signifié (pour soi) par la médiation des signes (parole-discours) n'en demeure pas moins toujours irrémédiablement au-delà du champ de la signifiance (transcendance) = ce qui marque par là même la finitude (métaphoricité-métonymicité) de toute parole (immanenceombres) = ce qui ne cesse par là même de nous entrainer dans la quête de l'in-é-dit (travail de la réminiscence) = l'inconscient, c'est-à-dire ce qui de la réalité fut déjà une fois contemplé (le réel) puis oublié (refoulé) et dont il faudrait se ressouvenir par la médiation des ombres (L'imaginaire) = ce dont la résurgence et l'irruption fait naître le doute, l'incertitude, le désarroi et l'interrogation sur la plausibilité des significations reçues (le Symbolique) = ce qui une fois remémoré ne saurait plus cesser de nous solliciter en regard du temps à venir = ce qui finalement s'avère inépuisable et inachevable $=$ l'Idée 
UNE CONTINUITÉ ÉPISTÉMOLOGIQUE DOUTEUSE MAIS NÉANMOINS TENTANTE :

DE PLATON À FREUD, À TRAVERS MARX

Je veux dire ici que Platon, tel que je le lis, sans en être tout à fait convaincu, eut dû en d'autres termes conduire directement, et sans que l'on en soit démesurément étonné, à Freud ou peut-être même à un Marx que j'imagine encore ignoré de nous ou demeuré, si l'on veut, à l'état fœtal. Car, pour l'un ou l'autre de ces trois philosophes, la conscience, loin d'être d'emblée donnée au départ dans sa belle et mirifique transparence d'essence, fut essentiellement contrainte, pour se trouver, de se créer elle-même, de pérégriner dans les couloirs labyrinthiques et obscurs de l'imaginaire, de l'illusion, du rêve ou de l'idéologie, se cherchant à travers les secousses et les œuvres objectivées mais, en cela même, vite aliénées et vite aliénantes, de sa propre histoire. «Comment sortir de sa névrose » vaut bien autant que de dire en effet «comment sortir de son aliénation ou de sa misère bourgeoise ou prolétaire ». Et «comment sortir de son aliénation» vaut bien autant que de dire « comment sortir de sa caverne et se départir de ces ombres si séduisantes et si rassurantes qui nous font prendre des vessies pour des lanternes»; et "comment accéder à son intégration, à son identité » vaut tout aussi bien que de dire enfin «comment accéder à la Cité idéale » ou, si l'on veut, à la société transparente et sans classes, débarrassée de l'intérêt égoïste et de l'illusion aussi vaine qu'évanescente de trouver son équilibre et son achèvement dans l'accumulation du capital ou dans la consommation ostentatoire des biens matériels ou symboliques: cumulation de signes en ce dernier cas, cumulation de symptômes dans l'autre, le tout nous renvoyant à une interrogation de toute façon sémiotique sur la condition humaine, communément partagée par nos trois philosophes. C'est que la question assurément, on le voit bien, n'a rien a priori d'idéaliste ou de matérialiste, l'intérêt de la question ne résidant pas ici dans le triomphe de la thèse sur l'antithèse, de l'âme sur le corps, ou vice-versa, mais plutôt dans l'approfondissement du sens que fait ou que ne fait pas la poursuite hyperbolique à l'infini de l'apaisement de toute forme humaine de contradiction ou de division. L'esprit de toute démarche philosophique, quelle soit idéaliste ou matérialiste est, à mon sens, toujours là. Car en toute démarche effective, ce qui est toujours visé c'est aussi par ailleurs ce que nous savons très bien être un rêve ou un absolu. 
Mais un absolu dont nous ne saurions assurément non plus nous passer tant et aussi longtemps que nous nous appréhendons nousmêmes comme sollicités par la dialectique du mouvement, du devenir et de l'bistoire. Car autrement, il n'y aurait plus qu'à régresser jusqu'à l'opportunisme du moment présent, ce qu'en fait nous n'aurions même pas à choisir puisqu'il s'agit là manifestement de notre nature la plus spontanée et la plus paresseuse et puisque ce n'est jamais au vent que l'on demande de tourner. On sait toujours en effet qu'il tournera dans un sens ou dans l'autre, la seule incertitude anodine qui nous soit dès lors consentie étant de savoir quand il tournera. Aussi, sous ce rapport de l'absolu, Marx, tout matérialiste qu'il était dans sa manière de dire les choses, dans sa métaphoricité personnelle, pourra assurément être cité au banc des accusés idéalistes sous l'inculpation d'avoir entretenu des espérances démesurées à l'endroit de la société communiste future, l'homme ne pouvant être si bon, dira-t-on, que dans la tête d'un illuminé. Sera-ce là d'ailleurs le jugement tranchant que l'histoire portera sur lui? Tout comme Hegel pourra assurément en contrepartie être taxé de matérialiste du fait d'avoir présumé qu'à travers la phénoménologie de l'Esprit c'est bien tout le poids et toute la concrétude de l'histoire qui se jouait, celle-ci devant finalement accoucher de sa forme absolue et on ne peut plus matérielle: l'État Prussien. Ce qu'il y a ici de central, tant chez Hegel que chez Marx, ce qui les réunit à juste titre, c'est sans doute de savoir que la lutte est au cour de toutes choses, qu'elle se joue dans la matière aussi bien que dans l'esprit, l'esprit ou la conscience n'étant en quelque sorte que l'expression risquée, tentée, de ce qui, au cœur de la première, demeure irrémédiablement vital, bien que muet et dépourvu de sens.

Que conclure de ce propos sinon que l'enjeu de toute réflexion philosophique se situe assurément ailleurs que dans ces combats d'allégeance qui nous font lire discrètement tous les philosophes, quels qu'il soient, sous la rubrique naïve de la philosophie de l'esprit ou de la philosophie de la matière, puisque réduits de la sorte à l'état d'anges ou de bêtes, d'esprit accidentellement incarnés ou de robots à intelligence artificielle, ils finissent irrémédiablement par nous paraître tous détestables et triviaux. Ainsi Épicure, dont le paradoxe le plus beau et le plus génial est de nous inviter à la conscience parfaitement mesurée de notre propre 
bien-être au milieu des jouissances et des plaisirs les plus raffinés, est-il classé contre toute prévisibilité, dans les archives désuètes de la philosophie, comme l'un des plus plats sensualistes qu'ait porté en son sein la civilisation occidentale. Il faudrait se convaincre ici que l'opposition matérialisme-idéalisme, outre le fait qu'elle soit infiniment trompeuse et ambiguë, n'est elle-même en réalité que l'un des symptômes de surface, bien réels pourtant, d'une contradiction plus fondamentale, plus viscérale, générique si l'on veut, anthropologique à la limite, que nous nous efforçons tous d'abolir, que ce soit par le biais de la cure de désintoxication philosophique ou par la cocaïne idéologique ou, si l'on préfère, entre les douleurs et les vapeurs. L'homme serait bien, en d'autres termes, et de naissance, un animal schizoïde, épris d'une passion aveugle pour les antinomies et les oppositions, et ne parlant toujours que dans les termes de la dominance de la Matière sur l'Esprit ou de l'Esprit sur la Matière, bien qu'en réalité son rêve le plus secret et le plus cher soit bien de se garder le privilège d'être les deux à la fois, de se les concilier et d'accéder à la jouissance qu'il pourrait retirer de se sentir pensée et de se penser sentant.

Il faudrait donc ici en arriver à se convaincre que Marx, tout matérialiste qu'il se soit lui-même déclaré, attribuait bien à la conscience, à l'idée, à la critique, à l'idéal, à l'idéologie ou à l'idole un pouvoir tout à fait réel de libération ou de purgation, ou encore un pouvoir de subjugation et de mystification, selon le cas, tout comme pour Freud dont on dit banalement qu'il aurait fait de la conscience un simple dérivé secondaire de la pulsion sexuelle, il est manifeste que le champ de la parole, de la talking cure si l'on veut, était bel et bien l'une des voies royales, bien qu'encore tout à fait incertaine, de la guérison. C'était reconnaître tout simplement que si au cour de la parole c'est bien toujours du corps et de ses conflits qu'il s'agit, que si au cœur de l'idéologie c'est bien toujours du corps social et de ses divisions que ça parle, il y a néanmoins dans le discours, dans l'ordre du sens, quelque chose qui se trame et se joue, qui ne saurait se jouer sur aucun autre terrain ou autrement, quelque chose donc de spécifique et de vital que l'on tenterait vainement de retrouver, comme tel, dans les replis cachés de l'infrastructure économique ou dans les voies synaptiques des neurones de notre cerveau. Ainsi on sait très bien par exemple que ce qu'on appelle les maladies du cerveau sont aussi et justement, 
dans bien des cas, des maladies de la communication humaine et qu'il ne suffit pas d'administrer des anti-dépresseurs ou d'autres produits chimiques similaires pour guérir des troubles de la représentation et du langage qui relèvent bien davantage de la violence symbolique. En d'autres termes, il y a plus d'une façon de penser les rapports du corps au monde. Et si la neuro-physiologie a tout le mérite de nous instruire sur les processus cellulaires et intercellulaires du corps cerveau, sans doute gagnerait-on à y associer conjointement une conception du corps comme corps symbolique, comme corps où se joue, à travers les mots et les affects que l'on y investit, la négociation psycho-sociale du désir ${ }^{4}$. Peut-on penser qu'il en irait de même de l'économie pour autant qu'il s'avère ardu de penser que le sens se jouerait quelque part dans la sphère de la superstructure et qu'il y aurait en bas, dans l'infrastructure économique, quelque réalité d'où le sens serait absent? À moins, bien sûr, que l'on soit déjà gagné à l'idée que l'économie est une simple affaire de distribution mathématique des biens matériels et de la force de travail, ou une simple équation mathématique du Capital, en sorte que la simple redistribution égalitaire des richesses serait une réponse à tous nos maux. Marx lui-même savait fort bien qu'il ne pouvait en aller ainsi. Aussi songea-t-il un jour qu' il faudrait peut-être en arriver conjointement à un homme nouveau, à une humanité désaliénée. C'était là une juste croyance car nous avons bien besoin de foi et d'espoir. Mais je ne sache pas qu'il nous ait quelque part garanti que la révolution socialiste de l'économie serait, à elle seule, garante d'une révolution dans la conscience humaine. Qu'entendait-il donc par révolution socialiste? Et quel était cet homme nouveau qui ne serait plus réductible à une simple force de travail? Il serait prudent de penser que sur ces questions épineuses qui concernent bien une anthropologie marxiste, Marx ne faisait qu'ouvrir le débat.

4. Cf. à ce propos le très beau développement consacré par Laing aux rapports entre l'approche psychiatrique-neurologique et l'approche communicationnelle dans Sagesse, Déraison et Folie, la fabrication d'un psycbiatre, éd. du Seuil, Paris, 1986, Pp. $150-158$. 
SUR LES CHEMINS NON PAVÉS D'UNE RÉFLEXION À INVENTER: OU À PROPOS D'UNE DIALECTIQUE DU CORPS ET DE LA PENSÉE SYMBOLIQUE

Notre passion pour les dichotomies et les antinomies insolubles ne s'étant guère dissipée depuis Descartes, on ne saurait s'étonner cependant que des penseurs aussi proéminents que Marx ou Freud aient été finalement accueillis sur les parvis ou dans les antichambres de notre culture sous les auspices quelque peu terroristes d'une véritable bérésie matérialiste mettant en péril les acquis moraux et intellectuels de l'occident tout comme Platon, évidé de résonance tragique et existentielle, avait été porté au nues de la chrétienté théologique et réduit plus souvent qu'à son tour à la fonction d'un simple fournisseur de concepts utiles pour penser la Transcendance. On ne saurait davantage s'étonner en conséquence que les disciples de Marx aient vite riposté à l'infamie en radicalisant les thèses matérialistes de celui qui était devenu leur saint patron en rendant clair et transparent ce qu'il pouvait y avoir encore d'obscur et d'incertain dans son œuvre. À la question, toute cartésienne dans sa formulation: "est-ce bien la conscience qui détermine la vie ou la vie qui détermine la conscience? », it appert finalement que Marx aurait répondu en optant résolument pour le second membre de l'alternative, nous confinant ainsi à une lecture purement épiphénoménale de tout ce que l'on peut entendre sous le dénominateur commun de culture, de pensée, de conscience ou de productions symboliques, et qu'il aurait ainsi transfiguré le champ de la conscience, voire même de la conscience de classe, jusqu'à n'être, en certaines versions exégétiques, que le reflet historico-social de l'intérêt économique régnant ou devant régner, selon les lois irréfragables de transformation de l'histoire. Comme si la conscience, parvenue enfin au faîte de la fatalité de ses illusions et de ses malheurs et délivrée des aléas de ses propres contradictions de classe, put enfin, à l'aube du communisme, se complaire dans toute la clarté de son innocence reconquise. Ce qui ne faisait guère mieux de Marx qu'un fonctionnaire petitbourgeois se donnant comme le négociateur attitré de la partie syndicale et devant désormais et tout aussi fatalement passer à l'avant-scène de l'histoire. "Posez le mouvement de la matière et vous aurez l'esprit » aurait pu être ici le mot d'ordre unanimement proclamé par tous les partisans résolument et irrévocablement gagnés au matérialisme mécaniste (quoique secrètement 
finaliste). Assurément ce matérialisme à la Taine ne valait guère mieux que de dire bêtement, d'une façon qui se réclamerait ellemême maladroitement et naïvement de Hegel: «posez le mouvement de l'idée et vous aurez la matière » (entendons le réel). Car, que l'on parte de la matière ou que l'on parte de l'idée, la question n'est jamais de savoir sur le mode binaire A/B quel terme détermine l'autre, mais plutôt de savoir comment $A$ peut entrer en relation avec $B$ de telle sorte que "quelque chose se passe » qui concerne à la fois $\mathrm{A}$ et $\mathrm{B}, \mathrm{B}$ n'étant plus ici qu'une certaine manière de penser relationnellement la réalité du devenir de $A$ et $A$ qu'une certaine manière de penser relationnellement la réalité du devenir de $\mathrm{B}$. Ce que Marx sut heureusement fort bien reconnaître et mettre au crédit de la dialectique quasi matérialiste du maître et de l'esclave. En d'autres termes, si l'on tient que «les idées dominantes de chaque époque sont celles de la classe dominante de son mode de production", la question qui se trouvera alors soulevée ne sera pas tant de savoir comment l'infra-structure économique détermine la superstructure des idées juridiques, politiques ou philosophiques, mais bien plutôt de savoir pourquoi sans superstructure tout court il ne pourrait même pas y avoir d'infrastructure, l'économie étant de facto une activité éminemment juridico-symbolique et ne pouvant être pensée autrement que comme un ensemble de pratiques matérielles signifiantes. En d'autres termes, la question qui se pose est bien de savoir d'où vient que le pouvoir économique ne peut se dire que du fait d'être médiatisé par le pouvoir du code juridique et symbolique et d'où vient que le pouvoir du code, du signifiant structuré, ne peut luimême se dire à son tour que du fait d'être médiatisé par l'économie toute pulsionnelle du corps désirant, le désir participant manifestement et de l'économie libidinale et de l'économie sociétale, et du corps et du sens, et du besoin et de la règle qui régit les modalités de sa satisfaction, et étant donc peut-être de ce fait cette mystérieuse charnière, cette formation articulaire qui nous éviterait et les pièges d'un idéalisme délirant et ceux tout autant insidieux d'un matérialisme bétonné.

FREUD ET LA QUESTION CENTRALE DU DÉSIR :

ENTRE LE CORPS ET LA CULTURE

La question du désir est donc ici assurément centrale et si on peut la faire remonter jusqu’à la tragédie d'Oedipe-Roi ou encore 
jusqu'au mythe de l'androgyne de Platon, puisque c'est bien là que se trouve déjà posée assez explicitement, pour la première fois peut-être, la triple question épineuse du manque, de la complémentarité narcissique et de la différence, c'est par ailleurs à Freud et à la psychanalyse que l'on doit de l'avoir posée de façon plus globale et plus centrale encore au niveau d'une triple articulation du corps, du signe et du pouvoir, corporéité et socialité se rejoignant sur le terrain d'un seul et même questionnement. Rappelons à cet effet que déjà dans l'esprit de Freud, et dans celui de l'Association psychanalytique internationale fondée en 1908, le but du développement des recherches psychanalytiques, bien que poursuivi surtout dans le cadre thérapeutique, devait consister à "cultiver et à faire avancer la science, soit en tant que psychologie, soit dans ses applications à la médecine et aux sciences humaines » 5 , la présomption de base étant que la connaissance des névroses et des psychoses, des comportements dits pathologiques, pouvait servir de base à la compréhension de la psyché et des comportements humains de façon générale. C'est dans cette perspective éminemment interdisciplinaire que Freud en arrivait d'ailleurs à conclure que « la sociologie qui étudie le comportement de l'homme au sein de la société ne saurait être autre chose que la psychologie appliquée.... ${ }^{6}$. Énoncé sans aucun doute restrictif dans sa formulation mais qui donnait à penser que les phénomènes économiques, politiques et culturels relevant même des plus hautes sphères de l'activité humaine ne sauraient être envisagés sans cette référence capitale au pulsionnel et à l'inconscient comme ce à partir de quoi s'élaborent les constructions les plus sublimes de l'esprit humain et ce sur quoi misent, comme sur l'énergie qui les alimente, l'ensemble des activités pratiques de l'homme. "Point de pulsion point de refoulement, point de refoulement point de désir, point de désir point de représentations d'objets et point de représentations d'objets point d'agir » auraiton pu alléguer ici, à moins que l'on ne fut d'emblée gagné à une anthropologie ratiocinante. «Il est inadmissible, écrivait Freud, de

5. Cf. l'ouvrage de Jeannine Chasseguet-SmiRgel, Pour une psychanalyse de l'art et de la créativité, Paris, Payor, P.B.P. ${ }^{\circ}$ 305, 1971, p. 8 ; je me suis largement inspiré de l'article intitulé "La présence d'une illusion » (Pp. 7-47) dans l'élaboration des quelques paragraphes qui suivent.

6. Cité par J. Chasseguet-Smirgel, op. cit., p. 20. 
négliger le rôle des facteurs psychologiques (entendond biopsychiques - A. P. - ) quand il s'agit des réactions d'êtres vivants humains. Non seulement ces facteurs participent à l'établissement des conditions économiques, mais encore ils déterminent ensuite tous les actes de l'homme, lesquels ne peuvent réagir qu'avec leur instinct de conservation, leur agressivité, leur soif d'amour, leur besoin de chercher le plaisir et de fuir le déplaisir » 7 . Le primat, si l'on veut, ainsi concédé à l'inconscient ne signifiait pas pour autant, aux yeux de Freud, une disqualification non plus qu'une minimisation des activités conscientes, toute symbolisation consciente (sublimation) ne s'entendant que du fait même de son articulation sur l'inconscient et l'adage clinique, existentiel ou humaniste de Freud demeurant toujours en tout état de cause « là où JE était, ÇA doit advenir ", l'impensé du corps, l'inexprimable, le refoulé, relayés par les constructions narcissiques du Moi, se trouvant en quelque sorte appelés ex voto à regagner ainsi le champ de la conscience. Freud visait tout simplement à signaler que toute activité humaine, individuelle ou collective, matérielle ou mentale, vise, par certains côtés, à satisfaire, à trouver des solutions à des besoins instinctuels, pulsionnels, émotifs, affectifs qui jouent à titre de motivations puissantes et souvent méconnues chez tout individu, fut-ce dans la conquête, dans l'exercice et dans la consolidation du pouvoir, dans les rapports amoureux, dans le plaisir des échanges et des communications inter-individuelles, dans les manifestations collectives et populaires, dans les actes d'agression belliqueux, dans les activités de création artistique, littéraire ou scientifique, dans le recours aux rites et aux cérémonies communautaires, dans la rêverie éveillée, dans l'élaboration des fantasmes inconscients et conscients aussi bien que dans les représentations oniriques.

Conséquemment, en postulant et en établissant un lien dynamique entre les activités bio-psychiques primaires et les élaborations secondaires de culture, en présumant que «si les névroses pouvaient apparaître comme des tentatives pour trouver des solutions individuelles compensatoires pour les désirs insatisfaits (c'est-à-dire des formes de réalisation symptômale et/ ou imaginaire du désir refoulé), les institutions sociales

7. Ibidem. 
cherchaient à fournir elles aussi des solutions sociales à ces mêmes problèmes", Freud se trouvait à rendre solidaires l'analyse des processus de la psyché et l'analyse des réponses socio-culturelles apportées historiquement aux besoins primaires et fondamentaux de l'homme: besoins organiques, certes (se nourrir, dormir, se reproduire, se protéger des stimuli douloureux ou désagréables), mais besoins organiques qui sont eux-mêmes indissociables des besoins de sécurité affective, d'autovalorisation, de communication émotionnelle, de réalisation de soi, d'expression et de créativité liés aux modalités sociales, formelles ou informelles, de satisfaction de ces besoins. Du point de vue de la psychanalyse, en effet ${ }^{8}$, l'homme est un tout, une totalité non réductible à l'une ou l'autre de ses dimensions (biologique, psychique, sociale), le domaine de l'inné étant indissociable du domaine de l'appris et, par conséquent, des figures et des mécanismes qui servent de médiation entre l'inné et l'appris, entre l'organisme et le milieu, entre la structuration de la personnalité individuelle et la structuration institutionnelle des groupes et des collectivités. En soulignant en conséquence qu'aucun système socio-politique ou socio-économique, ni capitaliste ni socialiste, ne pouvait être intrinsèquement envisagé comme une solution acquise et définitive aux problèmes humains ${ }^{9}$ puisque ce ne sont pas les systèmes en soi qui se trouvent à répondre aux problèmes humains mais bien les hommes concrets qui y œuvrent et qui sont euxmêmes aux prises avec leurs propres désirs et avec leurs aléas, Freud se trouvait à attirer l'attention sur la nécessité d'une analyse critique et concrète des formes historiques de sociabilité, de rapports individus/groupes sociaux, de rapports individuspratiques de socialisation. Concomittamment, il ouvrait aussi la porte à des applications pratiques de la psychanalyse hors du champ thérapeutique, ce qu'il fit lui-même d'abord, que ce soit dans le domaine de la psychanalyse de l'art, de la littérature ou de la culture. L'apport primordial de Freud consistait donc à fournir, dans la perspective limitée qui est propre à la psychanalyse, une compréhension en profondeur des faits sociaux, en insistant avant

8. $\grave{A}$ noter que je n'écris pas pour autant «du point de vue des psychanalystes», la psychanalyse, comme tout autre savoir pouvant, dans bien des cas, devenir une entreprise rétrograde et conservatrice.

9. Cf. Sigmund Freud, Malaise dans la civilisation. 
tout sur l'idée que toute manifestation de socialité, qu'elle soit de l'ordre de la création ou de la reproduction, de l'innovation ou de la résistance au changement, de l'économie individuelle ou de l'économie collective, repose sur une mise à profit des équilibres plaisirs-déplaisirs et ne devient compréhensible que par la mise en évidence des processus d'inscription des pensées et des désirs inconscients dans l'ordre de la symbolisation et de la représentation consciente, dans l'ordre donc à proprement parler de la culture.

\section{DE LA PULSION À LA RE-PRÉSENTATION : LA PENSÉE} COMME TRAVAIL DE SUBSTITUTION SYMBOLIQUE POUR MEUBLER LE VIDE DE L'ABSENCE

L'idée ou, si l'on préfère, l'intuition de départ qui est ici à la base des considérations de Freud est, à mon sens, fort simple bien qu'elle puisse s'avérer fort complexe dans ses développements et ses implications. Elle réside dans le fait que là où le corps, en état de besoin et de tension pulsionnelle se voit provisoirement privé de l'apaisement et du plaisir qui lui vient de l'objet extérieur de satisfaction anticipé ou attendu, c'est bien dans la réappropriation psychique de ce même objet de satisfaction sur le mode introjectif/ projectif de l'identification que le corps-sujet désirant se trouve en quelque sorte à meubler le vide de son absence, l'enfant trouvant dès lors dans le travail de symbolisation et dans son investissement pulsionnel une forme de réalisation et de satisfaction médiate ou détournée de son propre désir. On doit à Pierre Tap ${ }^{10}$ et plus récemment à Nicolas Duruz ${ }^{11}$ de nous avoir fourni des tableauxsynthèses des diverses théories de l'identification en nous en restituant le contenu essentiel en regard des différentes phases identificatoires (narcissique, primaire, œdipienne et postœedipienne), des différents types d'identification (de deuil, à l'agresseur, narcissique, de l'idéal du Moi, du projet), de la nature des investissements mis en jeu (libidinaux, non libidinaux, libido du moi, libido sexuelle d'objet...) et de leur importance

10. Pierre TAP, «L'identification et la genèse de la personnalité », in Annales, Université de Toulouse-le-Mirail, t. X, no 3 (1974), pp. 69-100; t. XI $n^{\circ} 3$ (1975), pp. 77-120.

11. Nicolas Duruz, Narcisse en quête de soi, Bruxelles, éd. Pierre Mardaga, Psychologie et sciences humaines, 1985 
relative dans la genèse et le développement de la personnalité normale et pathologique (ou l'entre-deux?). Chacun y allant en ce domaine de ses propres conceptions toutes relatives, on me permettra de souligner aux fins du présent article que c'est dans la théorie des imagos (identification primaire), développée par Mélanie Klein, que l'on trouve particulièrement bien explicitées les modalités de cette réappropriation substitutive du réel qui constituent, si l'on veut, la matrice première et générique de ce qu'on pourrait convenir d'appeler la pensée. Il n'est pas dans mon intention d'entrer ici dans le détail de cette théorie des imagos. On pourrait toutefois la résumer de façon succincte en disant que c'est par le biais du refoulement et des mécanismes subséquents du clivage de l'objet réel en objet bon introjecté (la mère incorporée génératrice du Moi idéal) et en objet mauvais éjecté et déplacé sur divers objets du milieu ambiant ${ }^{12}$, que l'enfant, dès son premier âge, en arrive non seulement à se prémunir contre l'expérience du déplaisir et contre ses propres tendances sadiques à détruire l'objet senti ou perçu (c'est selon les auteurs) comme objet distinct, mais aussi à « développer une tendance prédominante à la conservation de l'objet ${ }^{13}$, le clivage des imagos servant en quelque sorte à régulariser l'angoisse de l'ambivalence occasionnée par l'éloignement et l'absence provisoire de la mère et à la répartir sur la représentation en tant "qu'affectée d'un signe positif» et provisoirement satisfaisant puisqu'elle «permet justement le tri et la séparation de l'amour et de la haine » ${ }^{14}$. Partant donc de cette idée qui veut que le travail de substitution symbolique soit le fruit d'une défense contre les exigences pulsionnelles et l'angoisse (ce que Freud exprimait déjà d'une certaine manière dans la Traumdeutung en disant que, fondamentalement, c'est-à-dire quant à son fondement, "la pensée n'est pas autre chose que le substitut du désir hallucinatoire») aussi bien qu'un processus constituant de la psyché essentiel à l'émergence du moi et à sa maturation ultérieure, Martinon a très bien su exprimer cette intuition centrale de Freud à propos de la représentation en tant

12. Cf. Jean-M. Petot, Mélanie Klein, Paris, Dunod, coll. Psychismes, 1979, en particulier les pp. 301 à 315.

13. Cf. Idem, p. 312.

14. Ibidem. 
que fondée sur l'identification en la mettant étroitement en relation avec la genèse même de la fonction symbolique comme instance d'acculturation du désir. Ainsi, citant d'abord Freud, il écrit :

«"Les situations de satisfaction répétées ont créé l'objet 'mère', objet qui, absent et désiré au moment où le besoin est ressenti, subit un investissement intense qu'on doit nommer nostalgie." Et c'est à partir de cette nostalgie vis-à-vis de la mère que le désir passe le simple besoin. C'est à ce moment que s'instaurent des circuits symboliques ayant pour but d'exprimer ce qui ne peut être possédé... comme si l'on trouvait au niveau symbolique cette tentation de montrer d'une manière ambiguë la liaison s'établissant entre le dire et le nonpossédable. C'est dans le va-et-vient ininterrompu entre l'expression et la possession que les formes sublimées du désir existent en tant qu'objets de culture... Aussi toute réalité ne saurait être comprise qu'en relation avec le problème de l'altérité et du désir...»" ${ }^{15}$.

Que la représentation symbolique de l'objet soit ici fétiche, objet transitionnel ou encore objet intentionnel ne relève pas de mon propos immédiat. Car ce n'est point du statut épistémologique de l'illusion qu'il est ici question. Ce qui par contre relève bien de mon propos, c'est que toute construction symbolique d'objet, dans une perspective psychanaly tique, se trouve bien à reposer sur cette matrice initiale du non-possédable comme souche de tout processus identificatoire et de toute relation d'objet ultérieurs, ce que nous appelons la pensée, comme fonction de re-présentation, ne pouvant dès lors être considéré dans le processus même de sa genèse et de sa constitution que comme substitution métaphorique 16 par rapport à l'objet archaïque du désir. Ce qui fait dire à Gantheret que « le maternel... est le soutien implicite et muet de toute parole, de tout choix d'objet » et que « toute élection de signe est tentative

15. Jean-P. MARTINON, Les métamorphoses du désir et l'oeuvre, le texte d'Éros ou le corps perdu, Paris, éd. Klincksieck, 1970, pp. 26-27.

16. À propos de la corrélativité des dimensions métaphoriques et métonymique de la pensée cf. Albert Henry, Métonymie et métaphore, Paris, Klincksieck, 1971 ; E.K.K MARANDA, "Structure des énigmes", in Travaux inédits, Cahiers du CELAT, no 1 (1982), Université Laval, Québec, pp. 32-42; A. WILDEN, Système et structures, Montréal, Boréal Express, 1983; Guy Rosolato, Essai sur le symbolique, Paris, NRF, Gallimard, 1969. 
pour le restaurer et y échoue par son découpage même » ${ }^{17}$. En d'autres termes, l'expérience initiale du manque, de la séparation ou du délai de satisfaction comme expérience incontournable et fondatrice du processus même d'humanisation conduit nécessairement à la recherche d'un mode de satisfaction de type illusoire et fantasmatique qui est essentiel à l'établissement d'un équilibre homéostatique dans l'économie libidinale, c'est-à-dire dans la répartition et la décharge de l'énergie pulsionnelle sur l'organisme dans son rapport physique, sensoriel ou intentionnel au milieu. C'est sous ce rapport que Martinon se trouve d'ailleurs à postuler la solidarité fondamentale de la réalité représentée et du désir, la représentation symbolique, parce qu'elle est elle-même un lieu d'étayage des pulsions, pouvant être dite bel et bien corporelle tout comme, indistinctement, on pourrait tout aussi bien dire que le corps est lui-même ici infiniment symbolique. Ce serait d'ailleurs là une façon d'interpréter ce que Freud appelait pour sa part le représentant psychique de la pulsion.

Ainsi, en tant qu'être de besoins en regard d'un environnement avec lequel il se trouve en état forcé de discrétion, de pénurie, d'impuissance relative et, par conséquent de désir, l'homme, de par sa prématuration biologique (Bolk) et sa situation de dépendance particulièrement marquée, est manifestement dès l'origine un grand réalisateur de représentations, un grand quémandeur de métaphore, un insatiable metteur en scène de l'univers qui le contient puisque dans l'économie intentionnelle de son rapport au monde c'est bien toujours une visée de satisfaction, de plaisir et de réponse à son propre manque qu'il poursuit. On le remarquera en particulier à l'intensité avec laquelle l'enfant se livre très tôt à des constructions imaginaires dans ses activités ludiques, meublant son rapport au monde des fantasmes et de l'imagerie sur lesquels prend appui l'édification et la restauration permanente de son propre moi, jouant avec les sons et avec les mots qu'il apprend à y associer, investissant magiquement par le biais de ses monologues les objets réels qui le circonscrivent, les

17. F. Gantheret, Incertitude d'Éros, Paris, NRF, Gallimard, coll. Connaissance de l'Inconscient, 1984, p. 232. La problématique de l'absence y est traitée à partir de la page 228. Cf. aussi J.B. PonTalis, Entre le rêve et la douleur, Paris, Gallimard, coll. TEL, 1977, p. 197. 
transformant au gré de ses fantaisies ou de ses caprices et trouvant dans cette réalisation imaginaire du désir de quoi dissiper l'angoisse liée au refoulement de toute séparation, de toute absence, de toute castration, fut-elle méticuleusement et amoureusement mesurée et sainement symboligène ${ }^{18}$ ou ressentie au contraire comme une atteinte à sa sa propre intégrité.

En cela on peut dire dans la foulée de Mélanie Klein et à l'instar même de Martinon que toute « construction du monde sur le mode symbolique est liée à l'angoisse, parce que justement, dans l'expérience de l'angoisse [renaissante - A.P. - ] nous avons le sentiment que notre armature symbolique repose sur le vide et qu'en conséquence la croyance en notre sécurité est aléatoire ${ }^{19}$. C'est là non seulement le cas du rêve qui se trouve en quelque sorte à ex-primer de façon manifestement métaphorique ce qui du système perception-conscience a été vécu sur le mode de la tension, de la détresse ou d'un danger flou ou insoutenable, traumatisant si l'on préfère, mais c'est aussi vrai de l'ensemble des productions symboliques culturelles, mythiques, idéologiques, esthétiques ou même scientifiques qui ont pour but d'assurer à l'homme le contrôle symbolique, fictif ou objectivant, des choses, des personnes et des situations avec tout ce qu'elles peuvent véhiculer pour lui d'inconnu, de menace, de risque, de frustration ou de résistance en regard d'une expérience de plaisir satisfaisante. Ainsi, dans une perspective proprement analytique, on doit dire que la pensée au sens le plus large que peut avoir ce terme (image mentale, signe ou symbole) est toujours infiniment intéressée en ce que même le fait de tenir rigoureusement compte des particularités de l'objet réel et de ses contraintes se trouve luimême toujours inséparablement associé, par quelque biais, à la poursuite d'une expérience de satisfaction et de plaisir. Toute sublimation, créatrice de sens ou compulsive, épistémophilique ou compensatoire, s'avère en cela étroitement liée au processus même de l'élaboration, de la conservation et de la protection du moi. Même ce que nous nommons la folie pourrait se définir sous ce rapport non pas comme une abnégation de soi-même en regard

18. L'expression est de Françoise Dolto, in L'image inconsciente du corps, Paris, éd. du Seuil, 1984.

19. Jean-Pierre MARTINON, op. cit., p. 29. 
du principe de plaisir mais bien plutôt, au contraire, comme l'instauration d'un totalitarisme du principe du plaisir, la psychose n'étant en fait toujours que la tentative ultime et paradoxale de rétablir, en regard d'un milieu pathogène, un équilibre plus ou moins gravement perturbé dans l'économie libidinale. C'est d'ailleurs sous ce rapport d'abord économique que Freud considérait le délire, qui implique une levée du refoulement et une impossibilité provisoire de sublimer, comme une tentative de reconstruction et de guérison sur un mode hallucinatoire. Que la pensée soit donc, dans tous les cas, infiniment intéressée est sans doute aussi infiniment heureux. Car si à l'expérience esthétique ou scientifique n'était associé aucun bénéfice psychique de plaisir, comme au rêve érotique ou même cauchemardesque est associé le plaisir ou de s'y prélasser ou d'en sortir enfin... dans la satisfaction d'avoir pu imaginer la nature du péril, on ne voit guère ce qui pourrait pousser l'homme à manipuler des symboles, à penser, à ordonner, à inventorier, à inventer et à transformer la réalité de son propre milieu. On ne voit guère, en d'autres termes, comment la socialité, qu'elle soit économique, politique ou autre serait ellemême rendue possible et désirable. Ce que Marcuse a su d'ailleurs fort bien illustrer $a b a b$ surdo pour les conditions du travail ouvrier en milieu industriel capitaliste, le principe de productivité ou de rendement comme principe de réalité totalitaire venant littéralement occuper toute la scène de l'organisation de la vie quotidienne et ne laissant guère à l'ouvrier d'autre loisir, en guise de plaisir, que des compensations et des satisfactions foncièrement asociales et névrotiques. L'ouvrage de Lasch, consacré à la société narcissique nord-américaine contemporaine, société d'abondance, de chômage, d'ennui et de consommation hédoniste où l'imaginaire fantasmatique, comme actualisation compensatoire du principe de plaisir, tend à régner en roi et maître aurait sans doute plutôt tendance à démontrer qu'il s'agit maintenant de compensations psychoticisantes...

DE L'AMBIGUITÉ CONSTITUTIVE DE TOUT SUJET: OU ENTRE L'IMAGINAIRE ET LE SYMBOLIQUE

La seconde intuition qui soutient les considérations de Freud sur le rapport corps-culture, plus visible à partir de son ouvrage Pour introduire le narcissisme, est sans doute d'avoir pressenti à 
l'encontre de la métaphysique classique et de certaines versions psychanalytiques ultérieures qui rejoignent les conceptions de Hartmann et de Guntrip ${ }^{20}$, que le Moi, loin d'être réductible à une essence ou à une instance psychique préexistante dont la fonction en serait d'abord une de synthèse et de conciliation délibérante entre les pulsions du Ça, les interdits du Surmoi et la réalité extérieure, était en fait lui-même une construction élaborée sur la base des mécanismes identificatoires ${ }^{21}$ et qu'à ce titre le principe de plaisir et le principe de réalité, pour autant qu'ils se jouent non seulement sur la surface du corps érogène mais aussi dans le corps représentation-d'objet, ne pouvaient manifestement être compris eux-mêmes qu'à partir de leur indéfectible corrélation. Cela revenait à dire que le statut de l'espace mental à travers lequel se noue et se développe le Moi du sujet désirant ne peut s'entendre que de l'articulation de la dimension du symbolique sur la dimension de l'imaginaire, le Symbolique, comme aptitude acquise à se distancier et à discriminer ce qui relève du signifiant et ce qui relève du signifié, ce qui relève de la représentation d'objet et ce qui relève de l'objet réel représenté, portant toujours dans son flanc l'ombre de l'Imaginaire: c'est-à-dire cette tentation toujours virtuelle de prendre le mot pour la chose, d'attribuer au mot un certain pouvoir de se substituer au réel et de l'entraîner ainsi sur la voie de la déréalisation. Cette tentation se confirme assurément dans le cas de l'illusion qui dit pourtant à sa manière un certain rapport au réel. Mais cela peut se dire aussi de tout travail de symbolisation, poétique, philosophique ou scientifique, pour autant que toute fiction créatrice, qu'elle soit heuristique, imagée ou hyporhétique, renvoie toujours, par-delà l'objet visé et ainsi signifié, à l'univers psychique des formes et des jeux de langage qui se trouvent investis par le créateur. Toute démarche d'objectivation du réel implique toujours en ce sens une déréalisation préalable de l'objet, c'est-à-dire un travail de décomposition-recomposition du réel perçu qui se soutient luimême du regard que l'on porte sur lui. Or, et c'est ici que l'apport

20. Cf. J.B. PONTALIS, Entre le rêve et la douleur, Paris, Gallimard, coll. TEL, 1977, Pp. 174 175 .

21. Octave Mannoni, Clefs pour l'Imaginaire ou l'autre scène, Paris, éd. du Seuil, coll. Points, 1969, p. 171 : « Le lieu de l'Imaginaire, c'est le Moi, non pas celui des débuts de la théorie freudienne, qui était chargé d'assurer l'adaptation à la réalité. C'est au contraire le Moi du Narcissisme, le lieu des reflets et des identifications » 
de la réflexion analytique est capital, tout regard est bel et bien une œuvre du désir. Et si ce regard s'alimente des matériaux puisés dans la banque des connaissances acquises et des perceptions qui lui viennent de l'exploration plus ou moins méthodique de la réalité, il s'alimente tout autant des schèmes et des esquisses de l'imaginaire qui plonge ses racines dans le retour ainsi métaphorisé du refoulé. Tout travail de symbolisation implique en effet que l'on reporte dans la production de formes signifiantes projetées sur la réalité qui fait l'objet de notre préoccupation immédiate un désir plus fondamental de savoir et de trouver qui tient au fait que quelque chose en nous s'est irrémédiablement perdu sous le coup du refoulement et de la dénégation. Créer des connaissances implique donc non seulement le sentiment que nous ignorons quelque chose de l'objet mais aussi le sentiment ontologique d'un manque par rapport à tout objet virtuel, c'est-àdire, matriciellement, par rapport à l'Objet manquant constituant du désir comme désir de le retrouver ailleurs et autrement. On ne comprendrait pas autrement que toute création ou toute découverte scientifique, requiert toujours, du fait de son caractère éminemment subjectif, une épreuve de réalité, c'est-à-dire l'expérience souvent crisogène des résistances, des déceptions et des anomalies (Kuhn) que peut offrir le monde des objets réels au cours prospectif et constructiviste de la fonction symboliqueimageante et, par voie de conséquence, aux schèmes, aux esquisses mentales et aux paradigmes psychiquement investis (dans le cadre de la science normale et sanctionnée) à travers lesquels nous appréhendons ces objets. Sous ce rapport toute hypothèse scientifique ne vaut en effet, en ce qu'elle prend aussi appui dans l'imaginaire et dans la méconnaissance relative des limites d'un cadre ou d'un protocole expérimental familier que pour autant qu'elle ne peut pas encore être infirmée ou invalidée de quelque façon par quelque autre épreuve de réalité à venir. Et ce pas encore repose sans doute sur la ferme conviction que toute représentation est toujours passible de désuétude et d'éviction et qu'il faut en science comme en poésie et en peinture déployer beaucoup d'énergie à imaginer et à démultiplier les formes pour risquer d'en décrocher quelques-unes qui soient plus beureuses ou plus objectivantes que celles qui sont déjà données. En cela toute connaissance ne se dit bien que de la conscience de son caractère provisoire et approché (le Symbolique), toute révolution ou toute 
évolution dans la manipulation technique du réel et dans le champ des représentations symboliques possibles de ce qu'il peut être se chargeant bien toujours d'en révéler a posteriori les limites, les inadéquations, si ce n'est les aberrations.

Que la pensée soit donc ainsi toujours articulée de façon plus ' ou moins dialectisée sur la double dimension de l'Imaginaire et du Symbolique, cela tient non seulement au fait que la représentation comme sublimation manquée (compulsive) ou réussie (élaboratrice) ${ }^{22}$, et donc comme concomittante du désir, est toujours arrimée au refoulement et donc au non-dit et à l'impensable sur lequel elle fait précisément silence pour que puisse justement prendre place la substitution d'une représentation du réel, mais c'est aussi que la réalité, pour cette même raison, ne saurait jamais nous être livrée comme telle. Elle ne l'est toujours en fait qu'à travers le défilé des signifiants (les ombres de Platon?), l'objet n'étant jamais saisi et possédé intuitivement dans son être même mais bien toujours à travers une élaboration imagée ou symbolique qui implique qu'il soit d'abord nié comme altérité radicale pour qu'en contrepartie il puisse être réaffirmé à travers ce qu'il n'est pas (le mot en effet n'est pas la chose) et qui pourtant en tient lieu. Sous ce rapport, la pensée s'avère toujours être littéralement une passion ou une nécessité à laquelle nous ne saurions renoncer puisque c'est par elle que se rétablit l'équilibre de notre rapport corporel au monde. D'un côté nous y cherchons la satisfaction de notre besoin de connaître les choses comme distinctes de nous pour y avoir d'autant mieux accès que nous respectons cette distinction ${ }^{23}$ (principe de réalité), d'un autre côté nous y

22. Le terme sublimation est manifestement ambigu. $\grave{A}$ ce propos, W. ReIcH distingue la sublimation de la formation réactionnelle de la façon suivante : "L'un des critères de distinction est que la formation réactionnelle a un caractère compulsif et figé tandis que la sublimation est spontanée ». Ou encore : « La sublimation semble établir un lien direct entre le Ca et la réalité, en harmonie complète avec le Moi et le Moi Idéal ; en cas de formation réactionnelle, on dirait que tous les actes sont dictés à un Ca rebelle par un Surmoi rigoureux»; in L'analyse caractérielle, Payot, P.B.P. no 289, Paris, 1971, p. 171.

23. Gantheret définit l'épreuve de réalité en disant que «le jeu de la différence est l'opération même de la Realitätsprüfung qui l'engage sur deux fronts conjoints: différence entre l'image-souvenir et la perception d'un objet extérieur; différence entre cet objet, affirmé adéquat, et tout autre déclaré inadéquat», op. cit., p. 229. Conjoints me semble signifier ici que ces deux opérations, dans les faits, se recouvrent et n'en font qu'une. 
cherchons tout autant la satisfaction du plaisir que la réalité ne peut, dans une très large mesure, que nous refuser. Aussi toute l'économie psychique, en ce qui concerne la représentation, se jouait-elle bien aux yeux de Freud, entre deux pôles à la fois indissociables et antithétiques, et donc plus ou moins dialectisés :

1) soit celui de la capacité de tolérer l'ambiguité et le paradoxe incontournable des mots et des images que nous produisons des objets, ce qu'on appelle le Symbolique pouvant dès lors se définir comme cette aptitude plus ou moins heureusement élaborée à être sensible et attentif à la dimension métaphorique du langage, c'est-à-dire à ce qu'il devrait y avoir d'indétermination consciemment maintenue au coeur même de toute détermination symbolique de l'objet (les mots n'étant pas les choses, ils ne peuvent au mieux que tendre vers l'établissement d'une certaine détermination sans ne jamais pourtant pouvoir se clore sur eux-mêmes, le sujet parlant ne pouvant que pressentir indéfiniment les points de fuite ou d'ouverture de son propre discours en regard de la référence);

2) soit celui de l'Imaginaire qui renvoie non seulement au plaisir de penser et de dire le monde au gré de sa fantaisie mais aussi et justement à la tentation de prendre le mot pour la chose, de coller sur le mot, sur la détermination signifiante, sur le signe. Du strict point de vue de l'Imaginaire, en effet, non seulement la représentation se trouve-t-elle à décliner ici sa fonction référentielle mais elle se soutient dès lors indistinctement d'elle-même comme réalité d'objet. C'est là, à proprement parler, ce qu'on appelle communément rêver, que ce soit les yeux fermés, pour le plus grand bien-être du dormeur, ou les yeux grand ouverts, ce qui ne manque pas parfois de soulever les imbroglios les plus comiques ou les plus tragiques. En cela, qu'on le veuille ou non, les mots, les images et les symboles sont toujours un peu et parfois beaucoup, sous le coup de l'urgence, de l'occasion ou de la nécessité, nos enfants chéris, puisque d'une part ils sont bien de nous qui les émettons et de notre monde intérieur et puisque d'autre part nous nous y reconnaissons nousmêmes spéculairement (individuellement ou groupalement) 
bien avant que de les rapporter aux choses. Cela est vrai assurément du mensonge qui, par une sorte d'esquive, nous rend la situation moins bouillante ou moins désagréable (que nous l'alléguions dans la plus parfaite inconscience ou sous le coup d'une compulsion qui nous induit au remords mais dont nous constatons après coup n'avoir pu nous passer), mais cela est vrai aussi des simulacres de l'art et de la création littéraire (ou de toute autre parole), puisque de la passion du signe ou du simulacre, ainsi que je l'ai déjà dit, nous ne saurions jamais entièrement nous passer ${ }^{24}$. Comment en effet accéder sur la scène de l'Être sans ne repasser incessamment par les coulisses de l'apparence et sans investir les signes, sur un mode quasi-magique, d'un certain pouvoir de se substituer aux choses? N'est-ce point ce que peut nous révéler d'ailleurs la constatation a posteriori d'avoir été dupé par un vendeur d'illusion, de s'être abusé soi-même, ou encore celle d'avoir été saisi et transporté, sur un mode ludique et quasi-hypnotique, par une réalisation cinématographique particulièrement impressionnante? Que les mots puissent donc ainsi précéder, pour ainsi dire, notre rapport au réel est si vrai que même le scientifique sait très bien que, pour exorciser de l'imaginaire le champ de ses propres pratiques, il ne peut faire autrement que de recourir de toutes pièces à un langage technique susceptible de lui épargner les aléas et les ambiguités déroutantes de la polysémie ou les connotations trop personnelles ou trop régionales du langage ordinaire. Et pourtant même là, cette précaution ne réussit guère à lui épargner le recours à l'imaginaire et à la fiction, que ce soit dans une visée qui se veut essentiellement heuristique ou encore dans le fait que le scientifique étant un homme comme vous et moi, c'est-à-dire un sujet désirant, il est vraisemblable qu'il puisse se laisser aller lui aussi jusqu'à être séduit par ses propres paradigmes au point de les

24. Il ne faudrait pas en conclure pour autant que l'œuvre romanesque, par exemple, relève unilatéralement de l'imaginaire et qu'elle se trouve à évacuer par là le champ de la référence. Disons plutôt que le rapport de l'œeuvre à la réalité y est métaphorisé au sens où la psychanalyse entend ce terme. 
confondre avec la réalité et se montrer en cela étranger à toute critique ou hostile à toute théorie ou école de pensée adverse. De ce point de vue, la science, ainsi que l'a fort bien rapporté Bachelard, n'est jamais tout à fait coextensive avec l'esprit scientifique qui demeure toujours un idéal à poursuivre et à parfaire. Et sans doute la science gagne-t-elle toujours à se psychanalyser elle-même, ce qui fait bien partie de la formation de l'esprit scientifique. C'est que dans l'explication il y a toujours aussi une certaine passion pour les mots et les figures qui n'est pas sans relation avec les enjeux, les intérêts et les bénéfices investis de la chose (les bénéfices matériels étant par ailleurs toujours étroitement associés de mon point de vue aux bénéfices psychiques) mais qui tient tout autant, et parfois davantage, au caractère souple ou tranchant de la personne même du scientifique, c'est-à-dire précisément à la structuration caractérielle de son propre désir.

En cela toute représentation d'objet comme présenceabsence de l'objet réel, comme objet nié-réaffirmé, comme produit de l'univers psychique du sujet et de la réalité extérieure, serait bien à situer entre deux pôles tout au moins hypothétiques ou pensables: soit d'une part le pur fantasme qui sous-tend le discours et qui se fait passer pour plausible et pour vrai, pour plus réel que le réel lui-même (le fantasme comme défense étant restaurateur du principe de plaisir), soit d'autre part le mot, soutenu aussi par les formations du désir, mais qui ne cesse de se rappeler à son insuffisance dans la production d'une signification. En d'autres termes, il ne serait pas faux de dire que l'homme oscille, selon une infinité de modalités et de séquences temporelles particulières, entre l'illusion et la lucidité, entre la fantaisie et le petit quotidien, entre la rêverie errante et l'attention vigilante, entre un imaginaire débridé ou barricadé et un réalisme prudent bien que toujours capable aussi de rêver, d'imaginer et même d'inventer du réel. Sans doute s'agit-il là d'une nécessité impérative puisqu'à « refouler la fantaisie, [on finit toujours de toute façon par faire - A.P. - ] apparaître le fantastique » ${ }^{25}$. De ce même point de vue, on pourrait sans doute se risquer à dire aussi que ce

25. Octave ManNoni, op. cit., p. 113. 
que nous appelons communément la normalité ou la santé mentale, qu'il s'agisse de celles des individus, des groupes ou des collectivités, renvoie bien à cette capacité de tolérer l'angoisse de l'indétermination au cœur même de nos fixations inéluctables et nécessaires sur la détermination ou la vérité culturelles, à une disposition ou à une aptitude mentale à l'ouverture sur l'inédit, sur l'inachevable, sur l'indéterminé au coeur même du désir de dire et de signifier, d'identifier et donc de déterminer, créer du sens à propos de soi-même et du monde consistant dès lors précisément, ainsi que le disent si bien Ricardou et Barthes, à "différer continuellement l'expression» ou, si l'on préfère, à « inexprimer l'exprimable». Le réel pourrait se dire ici de ce trop plein de sens, de cet indicible qui fait infiniment de sens pourvu que l'on commence à en faire un peu à son propos et l'Imaginaire, concilié cette fois avec le Symbolique, de cette oscillation métaphorométonymique entre ce peu de sens qu'est la phrase comme énoncé parolier et ce trop plein de sens qu'est le visé encore inexprimé du réel, pour emprunter cette fois une expression heureuse de Rosolato ${ }^{26}$.

Ces considérations m'amènent finalement à penser que le $M o i$ freudien pourrait bien dès lors se définir lui-même, en empruntant cette fois à la terminologie winnicottienne, comme cet espace potentiel ${ }^{27}$ où le champ de l'illusion, comme espace mental de jeu avec les sons, les mots et les images, se trouve à déboucher, à des degrés variables et fluctuants, sur la dimension du Symbolique, tout sujet désirant ayant à composer entre une quête plus ou moins narcissisée de plaisir dans l'investissement pulsionnel des signifiants comme formes restauratrices d'un moi incessamment soumis à l'effraction plus ou moins mortifiante du réel et la prise en compte de l'altérité et de la différence de l'objet réel en tant qu'irréductible à la représentation ainsi investie de l'affect. C'est qu'au départ ce qui est donné ce n'est jamais d'emblée le réel mais bel et bien toujours une image heureuse ou malheureuse, bien que dans tous les cas supportable, de ce qu'il

26. Cf. Guy Rosolato, Essai sur le symbolique, Paris, NRF, Gallimard, 1969, p. 323.

27. À propos du concept d'espace potentiel (ou d'espace transitionnel), consulter WinNicotr, Jeu et réalité, Paris, Payot, 1975. Cf. aussi la présentation succincte de ce même concept par Pontalis, op. cit., pp 176-189. 
peut être. En sorte qu'être initié à la réalité doit bien se dire toujours, là encore, d'une épreuve de réalité, c'est-à-dire de l'apprentissage d'une aptitude à la dés-illusion qui ne soit point pour autant justement une perte d'illusions ou, si l'on préfère, une décompensation du désir de pouvoir générer de l'illusion: apprentissage donc, dans le maintien du plaisir de fabuler, d'une disposition à pressentir et à anticiper aussi la résistance du monde à toute forme stéréotypée ou sur-investie de représentation, apprentissage de la loi du réel aussi bien, dirais-je, que de la Loi (lacanienne) du Père, du refus de l'autre de se plier trop complaisamment à notre propre désir, du refus du réel de se couler trop parfaitement dans le moule de notre regard et de notre dire. Une telle disposition à tolérer ou à supporter sans déni la frustration, la séparation, l'écart, le sevrage, en un mot la castration comme processus fondateur du travail d'élaboration substitutive de la pensée, du symbole comme symbole, dépend dès lors manifestement du plaisir, non point absolu justement, mais plutôt satisfaisant (on pensera ici à la mère suffisamment bonne de Winnicort) que le sujet peut éprouver à expérimenter la réalité et à l'articuler au moyen des matériaux symboliques codés qui constituent eux-mêmes des objets à poursuivre, à convoiter et à acquérir. Or la réalité, il va sans dire, celle qui se donne toujours à signifier primordialement et qui s'avère en cela décisive dans la structuration de tout rapport ultérieur de l'ego au monde, c'est bien toujours celle de l'éducateur lui-même. À la fois cible première de la pulsion en tant qu'elle peut se définir comme "pleasure and object seeking" et objet intermédiaire entre l'enfant et la réalité environnementale, l'éducateur est aussi celui qui peut, en raison de sa position privilégiée comme objet primordial du désir, lui rendre désirables et les contraintes des premiers objets réels qu'il expérimente en sa présence et les lois mêmes du code linguistico-culturel. Voilà pourquoi on peut sans doute dire que toute création de sens comme auto-création de l'ego, comme conscience de soi, comme altérité et différence propre (self) est toujours corrélative à la capacité apprise de jouer avec les matériaux et les contraintes du code pour signifier l'autre comme interlocuteur et objet de son propre désir en ce qu'il peut justement offrir de résistance suffisante (mais pas trop) à la dimension narcissique du désir de l'enfant pour que celui-ci soit contraint de le formuler et donc de chercher (la demande 
impliquant et la sublimation et la reconnaissance d'autrui), sans pour autant n'être poussé jusqu'à l'abdication du plaisir de trouver. Car tout semble bien se jouer ici, selon l'aménagement même du désir de l'éducateur, entre l'identification éventuellement portée jusqu'à la con-fusion mimétique (le Même) et la différenciation éventuellement portée jusqu'à l'incongruité non-encodable du simple bruit, identification et différenciation, adhésion et écart, possession et résistance de l'objet ne se disant dans la perspective d'une éducation émancipatoire du désir que du mouvement de leur réciprocité dialectique. Être capable d'empathie, communiquer, échanger, dialoguer implique bien en effet et le premier et le second terme de ces couples, l'élaboration du moi se jouant toujours dans cet espace plus ou moins flexible et dynamique d'intersection entre l'imaginaire et le symbolique, là même où l'on pourrait parler aussi d'un jeu, d'un entre-deux ou d'un va-et-vient entre le sujet et l'objet, entre le corps et le code, entre la pulsion et l'institution ${ }^{28}$. C'est sans doute à la conjoncture socio-éducative, ainsi que j'y viendrai par le biais du concept de pouvoir, que revient le crédit ou le débit de faciliter ou d'entraver cette double articulation de la subjectivité désirante. Qu'il y ait manque ou pauvreté excessive d'objet de désir ou surprésence et surrépression d'objet de contrainte (pour employer un terme marcusien) ou viceversa, et par conséquent dans les quatre cas manque de plaisir satisfaisant, voilà bien qui peut expliquer en tout cas que l'Imaginaire puisse à l'occasion venir envahir tout l'espace potentiel : soit que le code soit alors assimilé et investi comme tel, sur un mode quasi magique et fétichiste, comme pur plaisir (compensatoire-stéréotypé) du signe, c'est-à-dire sans que ne soit mise à profit dans l'élaboration du sens l'expérience corporelle des limites et de la concrétude des objets poursuivis comme objets de désir et de satisfaction (ainsi, par exemple, on parlera alors de $L a$ Femme plutôt que des femmes que l'on croit tant soit peu réellement connaître); soit que faute de code structurant de la pensée, le désir soit reconduit à n'investir que le corps propre ou que le fantasme comme pure image, comme au-delà intangible et inexprimable de la parole, le sujet se rendant par là même étranger

28. Sur cette relation du corps au code, cf. en particulier D. ANZIEU, in Psychanalyse et langage, du corps à la parole, Paris, Dunod, 1977; aussi R. GoR, Le corps et le signe dans l'acte de parole, Paris, Dunod, 1978. 
à toute articulation et à toute communication possible du sens. Or, sans corps et donc sans désir, la parole, devenue littéralement impersonnelle, abstraite et décharnée (le corps étant réduit au mot) ne pourrait manifestement plus se dire que de la banalité d'un on dit, tandis que sans code et sans conventions, sans risque donc de s'objectiver, elle serait tout aussi incapable de se rendre présente à autrui pour en arriver à s'habiter elle-même. À ce titre, toute culture vivante se dit bien d'une expérience vécue qui vient animer le code ou d'un souffle de vie qui en fait un lieu de parole. En tant que conduction du désir dans l'ordre d'un sens possible de soi et des choses, toute création culturelle est aussi création de l'ego, c'est-à-dire expérience dialectique de restructuration symbolique du corps dans son rapport aux objets aussi bien que déstructuration relative des stéréotypes du code socio-culturel dans son rapport au corps désirant. Voilà sans doute pourquoi toute création peut se réclamer, du fait même de l'inscription du désir dans une production de formes inédites, d'une transgression. Transgression reçue ou refusée, transgression adulée ou méprisée, mais transgression en cela précisément qu'elle vient non seulement abolir un indicible silence mais aussi ébranler à sa mesure les significations reçues et sanctionnées et jeter un doute ou une dissension dans l'ordre du Même.

Je dirais donc que l'apprentissage de la dés-illusion correspond bien dès lors à ce qu'on pourrait appeler l'apprentissage de la nécessité aussi bien que la précarité intrinsèque de tout signe comme signifiant investi et toujours passible à ce titre d'être surinvesti comme moyen de suturer ou d'obturer l'expérience toute humaine de notre manque et de contrer l'angoisse liée au sentiment de notre ignorance, de notre limite et de notre incertitude. En cela le signe et le symptôme finissent toujours par se rejoindre pour autant que c'est aussi en eux que nous déposons notre préférence pour ne point savoir, tout comme c'est à travers eux en retour que, faisant en creux allusion à ce qui est ainsi tu, nous nous ressuscitons nous-mêmes dans le sentiment maintenu de notre plénitude et de notre pouvoir. L'idéologie, le discours publicitaire, la mantique populaire, la croyance aveugle, le roman à l'eau de rose, comme signes et symptômes justement de quelque chose qui se joue ailleurs, sur le terrain de l'inavouable, peuvent dès lors se réclamer bel et bien, au même titre que le rêve, du 
langage de l'inconscient. Sans doute faut-il aussi savoir consentirà cette illusion, à cette incontournable inconscience qui est aussi le lieu trop humain de notre finitude et de notre étrangeté pour nousmêmes, ne serait-ce que parce que l'Imaginaire, du fait qu'il supplée justement à ce qui de la réalité est aride et irrecevable, est peut-être aussi pour cette raison même ce qui rend possible la relance de notre acharnement à nous y mesurer. Saurions-nous d'ailleurs choisir entre le Vrai et le Faux, entre le Pur et l'Impur, entre la Science et l'Idéologie autrement que par un urgent besoin d'éternité, d'absolu et de réassurance? Une telle perspective binaire, qui est elle-même illusion dans l'Imaginaire, contrevient à une compréhension adéquate de la psyché individuelle et de la vie sociale des groupes en ce qu'elle présume que l'ordre de l'inconscient, dont l'expression est précisément celle de la métaphore et de la métonymie individuelles ou culturelles, puisse être d'un seul coup aboli au nom d'un discours parfaitement transparent dans la Vérité. Mais savoir, il faut sans doute se le rappeler aussi, n'en existe pas moins pour autant que c'est toujours savoir, à travers des simulations approchées, qu'on ne sait yamais vraiment tout à fait, c'est-à-dire savoir justement que le signe, qu'il soit ou non indéfiniment démultiplié, ne peut jamais en aucun cas être pris pour la chose de la même façon que la chose ne saurait jamais nous être donnée, quant à son sens, autrement que par le signe. Ce que Lacan appelle l'accès à l'ordre symbolique ne serait rien de plus, mais assurément rien de moins non plus que le rappel de cette banale évidence.

DE L'INCONTOURNABLE SOLIDARITÉ DU DÉSIR ET

DU POUVOIR : OU DE LA SOCIALITÉ COMME

ENTRE-DEUX PROBLÉMATIQUE ENTRE

LA PERSONNE ET LE PERSONNAGE

Que l'homme soit appelé depuis l'aube de son existence jusqu'à son crépuscule à chercher les mots et à inventer les formes qui le rendraient, de façon approchée et progressive, à la conscience de l'expérience qu'il fait, en son corps propre, de son propre vécu, de son histoire aussi bien que de l'univers ambiant qui le contient, voilà bien ce que nous confirment les premiers mots d'esprit et les premières esquisses de l'enfant, le développement exponentiel des connaissances scientifiques, les figurations 
ingénues ou ingénieuses issues de la création poétique et la quête critique du sens dans la réflexion philosophique. Qu'en contrepartie l'homme soit aussi livré très tôt et sans répit en pâture au pouvoir fallicieux des mots, à la soumission aveugle de la croyance et de l'opinion, voilà bien ce que nous confirment aussi le discours de la guerre, les préjugés et les stéréotypes culturels de toutes sortes, les idéologies racistes, la séduction politicienne et les sollicitations publicitaires pressantes. En cela, l'homme apprend ou n'apprend guère à délivrer sa propre parole et à accéder au risque et à la tolérance active du paradoxe de dire les choses. Initié à la luxuriance du langage, il s'y retrouve aussi assujetti ; éduqué au dialogue il s'y découvre aussi muet; sollicité à penser, il finit aussi parfois par y perdre son pouvoir de douter; pressé de créer et d'inventer, il arrive aussi qu'il ne puisse plus que répéter ; incité à risquer hardiment, l'appréhension le confine aussi à la confirmation, convié à la dispute et à la dissonance, l'adulation l'obnubile et le rend à la complaisance. Le mot de Freud se vérifie assurément: c'est que le moi est double ; il se possède et il s'ignore; il se rejoint et se démet, il se crée et il s'emprunte : real self/false self, pourraiton alléguer, bien que ces expressions ne soient certainement pas des plus heureuses, la personne ne pouvant en réalité se dire ici que de son immixtion ontologique au personnage et le personnage que de son immixtion à la personne, tous deux étant inextricablement - entrelacés comme en une passion d'être et de savoir qui se joue au cœur même d'un destin pulsionnel.

Mais pourquoi donc justement un destin pulsionnel? Pourquoi le névrosé, pourquoi le psychotique, pourquoi le borderline ou le caractériel, certes, mais pourquoi encore cette division $d u$ moi qui nous est communément familière, ce clivage entre le manifeste et le caché, entre le personnage et la personne, si ce n'est précisément qu'au cœur même du désir, qui est toujours désir de réponses à notre demande, se joue toujours aussi l'incontournable réalité du pouvoir sur le corps désirant? Le pouvoir tient assurément d'abord au fait que c'est l'autre - qui est lobjet même du désir de l'enfant - qui, tout puissant qu'il lui apparaît d'emblée, dispose aussi des matériaux et des voies déjà toutes tracées de la signifiance. C'est donc à prendre appui ou à prendre forme dans le regard aussi bien que dans le discours de l'autre que se repaît d'abord la réalité du désir. En cela l'enfant est 
littéralement une émanation du pouvoir de l'autre. Il est d'abord spectacle. Il est pour l'autre et par l'autre. Il est suspendu à ses yeux et à ses lèvres. Il est personnage. «Suis-je bien? » interroge-t-il en espérant la reconfirmation d'un premier plaisir éprouvé. «Suis-je mal ? » quête-t-il à rebours et du coin de l'œil en espérant le moins grand déplaisir à venir. L'éducateur, l'autre donc, est ici le maîtred'œuvre et le magicien des signifiants. De par le choix de ses mots et les intonations de sa voix le réel se confirme, s'éclaire ou bien se désagrège. On ne saurait imaginer plus spectaculaire scénario de création ou d'annihilation. Le sens du monde se fait et se défait au gré des principaux protagonistes. L'enfant, lui, ne cesse de chercher à se créer tout en étant appelé à figurer dans ce scénario, en même temps qu'il se familiarise aux jeux de rôles. Et pourtant les magiciens et les maîtres du jeu et du sens ont bien eux aussi leurs désirs. Et par conséquent, pour d'autres qu'eux-mêmes, ils sont aussi incessamment en quête de personnages à jouer. Et il en va vraisemblablement de même indéfiniment et de proche en proche, le pouvoir social, dans sa forme primaire, élémentaire et irréductible, pouvant se dire de cette réverbération spéculaire à l'infini des désirs, de cette solidarité et de cette interdépendance universelle des désirs comme Désir d'être pour autrui, pour et par quelqu'un d'autre que soi-même. En sorte que finalement c'est à se reconnaître dans un faisceau d'images kaléidoscopiques démultipliées mais à toute fin pratique toujours convergentes que chacun est à la fois confirmation et confirmé de l'autre. Saurions-nous nous suffire parfaitement nous-mêmes et cesser radicalement de quêter notre sens dans les mots et les yeux de ceux que l'on désire? Saurions-nous, pour accéder à l'élaboration du sens que l'on peut faire pour soi-même, nous épargner les mille et un détours et les mille et un personnages auxquels ne cesse de nous convier le travail assidu d'acculturation du Désir?

Ce n'est donc point à vrai dire d'avoir ou d'être un, plusieurs ou de nombreux personnages qui fasse ici problème. Car chacun sait que l'ordre du pouvoir et de l'organisation du désir « traverse bien de part en part une société " ${ }^{29}$ et qu'aucune société ne saurait pourvoir à sa propre conservation et à sa propre reproduction si

29. Cf. Eliseo Veron, «Semiosis de l'idéologique et du pouvoir", in Communications, $n^{\circ} 28,1975$, P. 9 . 
l'on ne convenait, sous le coup même d'une contrainte qui vise à se rendre culturellement désirable, des rôles qui doivent régir et rendre nos rapports prévisibles. C'est ainsi que toute société comporte ses règles et ses procédures, ses croyances collectives et ses mythes, ses rites et ses cérémonies, ses cultes et ses modes, ses traditions et ses coutumes, ses protocoles de toutes sortes, ses formes de loisirs, ses mours et ses lois, ses distinctions de rang et de fonction aussi bien que ses fêtes populaires, ses carnavals et ses marginalités consacrées ou tolérées. Sorte d'unification illusoire dans la re-présentation si l'on veut, qui tend précisément à abolir la perception des différences trop singulières et qui, en rendant possible l'anticipation commune et partagée des attentes, des valeurs, des jugements et des comportements, rend du même coup possible la co-existence des individus et l'entreprise de travaux communs essentiels à la satisfaction des besoins humains. Le lien social est minimalement à ce prix : il implique toujours, si on peut s'exprimer ainsi, le leurre d'une identification constituante de la communauté socio-culturelle et ce leurre serait-il inexistant ou dissous, nous serions vraisemblablement contraints de le réinventer. Car ne plus pouvoir parvenir à le maintenir ou à le restaurer signifierait manifestement notre perte; il n'est pas de sujet désirant en effet qui puisse prétendre se passer de désirer le désir de l'autre, de se perdre tant soit peu dans son discours emprunté, et par là même de se concilier son concours, toute révolution, sous ce rapport, n'étant elle-même que la tentative devenue irrépressible de voir renaître ce désir sous la forme d'Éros là où il se trouve déjà sur la voie d'une extinction mortifère.

Que la socialité doive donc miser incontestablement sur la fonction de l'illusion qui soutient symboliquement notre sentiment d'appartenance, voilà bien qui milite en faveur de la reconnaissance de l'illusion comme partie intégrante et constituante de la réalité sociale. C'est d'ailleurs en cela même que la culture est le ciment de la société. Dès lors s'il est vrai que chacun de nous cherche bien à se dire, à se découvrir et à se créer dans l'expression langagière de l'irréductibilité de son vécu et de ses différences (émotions, perceptions, sensations, sentiments, représentations, idées, goûts, expériences, découvertes, explorations, rêves), c'est aussi toujours à se reconnaître soi-même dans le regard et le dire d'autrui que se trouve toujours déjà initié notre 
désir. Double mouvement paradoxal et pourtant complémentaire, souvent contradictoire, où le groupe se définit donc par l'individu et l'individu par le groupe, la personne par le personnage, le personnage par la personne, l'un ne pouvant aller sans l'autre et l'autre sans l'un. C'est ainsi que voulant $m e$ rendre présent à autrui c'est bien aussi à travers ses mots que je me donne moi-même à penser pour lui, ses mots renvoyant manifestement à leur tour en écho à ceux de celui ou de celle à qui l'autre a lui-même tenté de se rendre présent. Qui n'a point un jour ou l'autre fait d'expérience la découverte que les mots finissent ainsi irrémédiablement par nous échapper et parfois même par nous perdre puisqu'à peine proférés ils sont aussi parfois le lieu de notre propre dépossession? Qui n'a point fait d'expérience la découverte que tout risque de parole est aussi une occasion de méprise ? Cette déception n'est manifestement possible que parce que nous cédons chroniquement à l'illusion d'une parfaite symétrie ou d'une parfaite transparence des rapports humains aussi bien que du langage, la culture faisant justement figure de conteneur, de cadre ou de matrice identificatoire illusoire où chaque sujet humain, parce qu'il est lui-même sous ce rapport une Gestalt, se trouve en quelque sorte invité à venir d'abord cueillir la confirmation spéculaire de sa propre existence symbolique pour-autrui et à y déposer l'angoisse d'une impensable déréliction. En ce sens toute culture se dit bien d'un code ou d'un cadre socio-culturel, c'est-à-dire d'un système de sens qui permet aux individus d'une collectivité historiquement située

1) de communiquer entre eux en se référant à un réseau de significations socio-culturelles communes, c'est-à-dire à un réseau de signifiants/signifiés communs (l'imaginaire social);

2) d'orienter leurs actions selon les règles, normes, jugements ayant trait aux valeurs attribuées aux objets, personnes, actions, situations auxquels il est fait référence dans le champ symbolique (et en ce sens toute action, en tant que signifiante ou donnant prise à une signification, serait confirmation ou transgression, sanction ou écart en regard d'un code socio-culturel);

3) d'accéder ainsi à une identité groupale, c'est-à-dire à une perception de ce qu'ils sont et de ce qu'est le monde, identité par laquelle ils se trouvent instaurés dans la 
représentation comme sujets sociaux, comme individus psychiquement solidaires d'un groupe dont ils reproduisent et élaborent le code en vue de leur re-connaissance d'autrui et de leur propre re-connaissance par autrui.

Sans ce fond préalable et relativement permanent d'identifications spéculaires, il est manifeste que nous ne saurions non plus accéder à l'acquisition des connaissances et des savoir-faire hérités de la tradition et des pratiques sociales techniques et qui sont essentiels à notre survie biologique autant qu'à notre développement psychique et intellectuel. La culture comme référence spéculaire fait toujours figure de matrice en laquelle se noue la possibilité même de tout processus consécutif d'individuation, d'apprentissage, de création et même d'auto-création.

Or, s'il y a toujours nécessité pour chaque communauté et chaque organisation culturelle de poursuivre et de refaçonner constamment son identité groupale et si cette nécessité prend ellemême appui sur la subjectivité désirante comme désir du désir de l'autre, il y a bien aussi pour chaque sujet désirant le désir tout aussi légitime, bien que parfois moins évident, de ne point succomber à l'attrait de l'image qui lui est renvoyée de lui-même par autrui puisqu'une telle capture du désir signifierait assurément l'apogée d'un culte voué à la soumission. Aussi la question du pouvoir $s$ 'avère-t-elle ici centrale puisque c'est toujours en elle que se joue la négociation et l'issue plus ou moins médiatisée de cette ambivalence entre le désir du groupe et le désir de soi-même, entre l'identité groupale et l'identité personnelle, entre le personnage et la personne. C'est que le pouvoir est ici indissociable de l'avènement (ou du non-avènement) de toute parole pleine au même titre que toute parole pleine est elle-même indissociable de son propre rapport au pouvoir du groupe et au pouvoir du code.

Ce qui se trouve donc ici mis en jeu, ce n'est pas tant la réalité du pouvoir mais bien plutôt son ambiguïté. Car si le pouvoir social, tel que je l'ai précédemment défini, ne semble pas en soi faire problème, s'il s'avère être une nécessité constituante de tout sujet et de toute collectivité puisque de personnages et de jeux de personnages nous ne saurions encore ici manifestement nous passer, ce qui par contre pourrait s'avérer problématique, c'est bien plutôt, me semble-t-il, que l'on se sente contraint de ne plus 
pouvoir que jouer au point d'en perdre même jusqu'au plaisir de jouer. Car, dans ce cas, le jeu ne consisterait plus tant à fabuler pour éprouver le plaisir de se découvrir et de s'inventer à travers le désir du désir de l'autre (playing), mais bien plutôt d'être pris au filet d'une fabulation préexistante (a game) qui ne donnerait plus guère de marge de manœuvre à l'errance créatrice du désir. Ce serait alors, à proprement parler, «être pris ou piégé dans les règles du jeu».

LE POUVOIR COMME PÉDAGOGIE DE L'ENCODAGE :

OU À PROPOS D'UNE DOUBLE MÉPRISE

SUR LE DÉSIR ET SUR LE SENS

C'est que le pouvoir en soi non seulement ne suffit pas mais en réalité n'existe pas «; il lui faut toujours l'assistance d'une pédagogie. On en reconnaît généralement deux : celle qui se range du côté du primat de l'identité groupale (au risque de priver le personnage de sa personne) et celle qui promeut l'épanouissement de l'identité personnelle (au risque de priver la personne de son personnage). La première se voue à la socialisation du désir, la seconde à son émancipation. La première se voudrait collectiviste et la seconde personnaliste. L'État se confronte ici à l'artiste, le Tout organisateur à la partie marginaliste. Il faut bien admettre ici que cette nomenclature binaire n'est assurément que rhétorique mais c'est peut-être pour mieux en révéler les enjeux. C'est qu'à la base de ces deux formules pédagogiques, qui concernent bien la pédagogie du pouvoir, il y a, me semble-t-il, une double méprise : la première porte de toute évidence sur le sens du désir tandis que la seconde porte plutôt, dirais-je, sur le sens du sens. Dans le cadre de ces deux formules, le désir, en effet, se dirait univoquement de l'individu tandis que le sens serait d'emblée du ressort de la culture et du groupe. Ainsi serions-nous conviés à choisir entre l'équilibre et l'harmonie du pluriel et la jouissance du singulier, entre la Raison sociale et le caprice égoïste, entre la nécessité du public et le hasard du privé.

Il y a là, dis-je bien, une double méprise. Car si le désir est bien de l'ordre du corps et donc de l'individu, il est tout autant de l'ordre du verbe et de la parole, ce qui met doublement en cause le rapport de soi-même à autrui et le rapport d'autrui à soi-même. Il faut assurément savoir maltraiter Freud pour sabrer à l'horizontale et 
pour en arriver à penser autrement : c'est-à-dire à réduire le désir à une simple question de libido. C'est que le désir est manque et par conséquent élan vers ce qui pourrait lui répondre. C'est en cela qu'il se forme et qu'il s'éduque, que ce soit pour se fixer de façon passive et stagnante dans sa soumission à un Idéal indépassable, inaccessible et quasi-mythique, ou pour s'activer agilement dans une forme signifiante qu'il crée à ses risques et périls pour mieux s'y découvrir dans l'infinité de ses similitudes et de ses différences. On sait assurément que la mère et le père y sont pour quelque chose. Mais, par-delà la fermeture de ce familialisme, c'est aussi la culture elle-même qui se trouve interpellée dans sa globalité puisque, en tant qu'œuvre de pouvoir, il est bien de sa visée de se rendre présente au coeur de tout désir : en d'autres termes, le père et la mère ont aussi leurs éducateurs et on peut dire que de nos jours ils ont tendance à se démultiplier (mass media) et à se disputer les élans et les ébats du désir. Le désir est donc social et relationnel, il ne peut être pensé autrement qu'en situation.

Et il en va de même encore en ce qui concerne le sens. Car rien n'est moins sûr en effet que le sens du réel se trouve d'emblée entreposé dans les silos de la culture à l'état de simple disponibilité instrumentale, en sorte que le sujet désirant, posé à l'état d'extériorité par rapport à lui, n'en serait en quelque sorte que l'usager. L'idée d'une subjectivité désirante précédant ou transcendant le code culturel et son histoire et en faisant usage de façon délibérée par simple apprentissage de ses règles syntaxiques, un peu à la manière d'un écolier qui s'initie au maniement d'un code informatique, est ici manifestement aussi impertinente, que son envers selon lequel le sujet parlant ne serait lui-même que le code ou, si l'on veut, que le support du code, n'ayant d'existence et de spécificité symbolique que par lui. Double réductionnisme en effet de la notion de sens, la première version nous ramenant ni plus ni moins sur le terrain d'un réalisme naïf où, entre le sujet et l'objet perçu, il y aurait le mot à utiliser pour désigner adéquatement l'objet et où l'erreur ne pourrait incomber qu'à un mauvais usage de la langue ou qu'à une distorsion accidentelle ou menteuse du sens des mots, la seconde version faisant par ailleurs des significations culturelles codées la totalité du sens social possible de l'expérience du réel, comme si le code linguisticoculturel, ayant par lui-même trouvé quelque préséance à la 
manière d'une métonymie fétichiste ${ }^{30}$, pouvait se situer en marge de son histoire et de sa fonction pratico-sociale et en marge de l'historicité psychique du sujet qui parle : non seulement se trouvet-on à nier ici le contexte et les conditions historico-sociales de sa génération ou de sa reproduction, mais on se trouve tout autant à désavouer l'ordre du manque et du désir qui s'y investit dans la tentation, dans la tentative, dans la nécessité même où se trouve tout sujet désirant d'élaborer ses propres relations d'objet. Or, s'il ne peut justement en aller ainsi, c'est manifestement que la production du sens, qui implique le code, est toujours elle-même indissociable de la dimension du désir aussi bien que de celle du pouvoir sur lesquelles elle ne cesse aussi de s'articuler. En sorte que là où le pouvoir est en crise, le désir ne manque généralement pas de l'être aussi et que là où le désir, en crise, n'arrive plus à s'articuler sur le code, c'est que le code n'arrive plus lui-même à faire du sens, c'est-à-dire à mobiliser activement les énergies désirantes. C'est qualors le sens du monde, comme lieu de convergence du désir et du pouvoir, est à réinventer. Ou il suffit de changer le sens, ou il faut aussi changer le monde. Dans les deux cas, il faut arriver à expérimenter ce dernier autrement. Et dans les deux cas, la culture, comme œuvre conjointe de désir et de pouvoir, ne survit et ne se régénère qu'en sachant consentir à la mort de ses formes périmées.

\section{DU POUVOIR COMME POUVOIR OU IMPUISSANCE D'ENCODER LE SUJET DÉSIRANT : OU LES JEUX DU POUVOIR, DE L'AVOIR ET DE LA CONJONCTURE}

J'en viens donc à l'idée que tout pouvoir, socialement parlant, se dit donc bien du pouvoir d'encoder le sujet désirant, toute création, toute différenciation ou toute individuation culturelles ne pouvant s'effectuer sous ce rapport que sur le fond préalable d'une identification à ce dont il nous est dès lors loisible justement de

30. Par métonymie j’entends ici non seulement la dimension syntagmatique du discours culturel (au sens très large du terme) mais aussi et surtout toute polarisation culturelle du sens qui impliquerait, dans l'Imaginaire collectif une connotation, pourrait-on dire, fétichiste : fétichisme du signe linguistique, de la monnaie, de la valeur morale, de la marchandise, du maquillage, de l'idéologie, du totem comme objets partiels valant pour le Tout. Au sens donc que lui attribue Baudrillard dans son approche de l'idéologie (cf. «La réduction sémiologique », Nouvelle revue de psychanalyse, $\mathrm{n}^{\circ} 2$, 1970). 
nous démarquer, de la même façon, dirais-je, que le Symbolique peut se démarquer dialectiquement de l'Imaginaire, en un rapport de va-et-vient. Assurément le pouvoir social, ainsi entendu, ne se soutient bien concrètement que du pouvoir que l'on détient aussi sur les ressources monétaires et techniques, aussi bien que sur les compétences, les qualifications et les savoir-faire pratiques qui donnent accès à la fabrication, à la gestion, à la diffusion et à l'inculcation du code. En cela le pouvoir se fait politique. Et si, par quelque biais, nous disposons bien tous de façon parcellaire ou associative d'un certain pouvoir de faire jouer le code, de le fractionner, de le fissurer, de le démultiplier, de l'enrichir ou de le spécifier selon la nature et le lieu institutionnel de nos propres pratiques (famille, école, entreprise, groupe socio-professionnel, communauté religieuse, association, parti politique, etc.), il n'est point dit pour autant qu'il soit laissé à notre discrétion de transcender sereinement le champ global de l'organisation et de la distribution sociale des rôles, qui toujours nous précède, non plus que les luttes, les alliances, les solidarités et les confrontations autour desquelles se nouent la conquête du pouvoir et la maîtrise du champ symbolique. Tout pouvoir parcellaire s'inscrit bien à ce titre dans le cours de l'intérêt et des contraintes d'un réseau de micro-pouvoirs de la même façon que toute praxis symbolique $s^{\prime}$ inscrit bien elle aussi dans les règles $d u$ jeu qui structurent les rapports sociaux et dans la dialectique des conflits de pouvoir qui se trouvent à les modifier. Solliciter le désir, le socialiser, le capter dans un régime de représentations et de significations, c'est donc bien toujours le faire dans le contexte d'une conjoncture, avec ce qu'elle peut contenir de conditions favorables ou de résistances à toute stratégie d'intervention ou à toute pédagogie émancipatoire visant aussi bien la collectivité que les individus qui en font partie.

La culture, en ce sens, n'est jamais un lieu de tranquille identité non plus qu'une cuvre permanente de création authentique, d'intégration du moi personnage et du moi personnel. Et cela est d'autant plus vrai pour les sociétés contemporaines en proie aux réformes et aux réajustements technologiques chroniques ou en proie à des revirements concurentiels imprévisibles, ou tout au moins imprévus, sur la carte de l'économie ou de la politique nationales aussi bien que mondiales: famines, réalignements des échanges et des alliances commerciales, 
insolvabilité des dettes nationales, guerres, sursaturation des marchés, carences ou difficultés conjoncturelles d'approvisionnement en matières premières, manque de planification, corruption administrative, bouleversement du milieu écologique, répartition scandaleuse des richesses, des revenus et des biens de production et de consommation, chômage, répression politique et culturelle des minorités ethniques aussi bien que des classes laborieuses, sursaturation des media par une propagande et une publicité éclatée ou sans vergogne... Autant d'éléments et de facteurs de déstabilisation sociale qui affectent la crédibilité des systèmes de valeurs et de légitimation symbolique et qui perturbent la stabilité relative des repères essentiels au maintien du sentiment d'appartenance et d'identité groupales et collectives. Tel un organisme vivant dans un milieu soumis à des variations climatiques et écologiques tantôt progressives et récurrentes, tantôt accélérées et brutales, toute culture connaît ses temps d'immunité forts et vigoureux et ses temps de morbidité infectieuse, ses temps d'autorité et ses temps de fragilité, ses temps d'expansion et de croissance et ses temps de stagnation ou d'immobilisme, ses temps de fragmentation et ses temps d'unification, ses temps d'euphorie collective et ses temps de crise et de dislocation, ses temps de mort et ses temps de recréation. Sous ce rapport, on pourrait comparer la vie des organisations culturelles à celle des organisations psychiques individuelles, celles-ci se trouvant à osciller entre l'angoisse dépressive qui marque le crépuscule et la perte de crédibilité de ses modèles et de ses formes identificatoires révolues ou en voie d'extinction (là où se creusent le vide et l'absence propres au doute et à l'incertitude des temps présents aussi bien que l'enthousiasme encore inquiet qui prélude aux grandes reconstructions, aux initiatives et aux créations inédites, à l'établissement des croyances nouvelles) et l'euphorie quelque peu maniaque qui conduit à surestimer ses moyens de façon triomphante et à méconnaître ainsi ses potentialités concrètes et bien réelles. Entre ces deux pôles, l'immobilisme qui favorise une sécurité indifférente bien que souterrainement fragile. Toute culture suit bien ou précède en cela les transformations qui ont cours dans l'organisation ou la désorganisation du corps social. Elle ne cesse de se remodeler selon les jeux de force qui ébranlent ou consolident les assises des pouvoirs économiques aussi bien que politiques. Et si l'on peut se 
sentir parfaitement à l'aise dans sa culture au point de s'y engloutir dans un conformisme indélogeable, le désir s'y trouvant parfaitement capté et apaisé en deçà ou par-delà toute inquiétude raisonnable, il arrive aussi qu'on puisse ne plus s'y reconnaître. Serait-ce précisément que, ne pouvant plus rejoindre le désir, le pouvoir, devenu impuissant, ne réussirait plus aussi à l'encoder, l'abandonnant dès lors au jeu imprévisible de ses propres vertiges? Fous, criminels, suicidaires, vieillards isolés, drogués chroniques, délinquants, marginaux de toutes sortes, tous éventuellement, à court ou à long terme, étrangers potentiels dans leur propre culture, incapables de la suivre, de participer à son devenir et même de la contester. Pour eux tous, ce qu'on appelle l'identité culturelle ne serait en réalité qu'un bateau ivre, qu'un vaisseau fantôme sans quais et sans amarres. Errer serait ici n'avoir aucun port, aucune préférence, aucune référence fiable et crédible, aucune adresse sentimentale. Tandis que pour d'autres le code, exercé ou subi et adulé, serait sans doute tout, même au dépend des protestations et des secousses du désir qui s'y investit : c'est-à-dire un port aux mille navettes amarrées mais incapables de prendre la mer. Univers stable et doré, bien que morne et mort, et donc sans risque de naufrage. Univers répétitif d'un désir figé par la peur d'ex-plorer et de voir jaillir le sens du cours tangible de la vie réelle.

DU POUVOIR, DU SENS ET DU DÉSIR:

OU DE LA RÉPÉTITION À LA CRÉATION DE SOI

AU COEUR DE L'AMBIGUITÉ DE TOUTE

EXPÉRIENCE SOCIO-CULTURELLE

Pouvoir-discours-désir: jeux de pouvoir-jeux de discoursjeux de désir que, par esprit analytique, il serait tentant de bien distinguer et qui pourtant, on l'a vu, se jouent simultanément en un même espace. L'espace de la parole, des signifiants parlés, est toujours en effet celui d'au moins deux interlocuteurs (dont l'un peut en réalité n'être que fantasme), présents «en verbe et en corps » (en désir) dans la représentation et qui parlent d'un lieu qui leur est propre, d'une position de pouvoir (de savoir) qui leur est assignée (même imaginairement) et qui est celle d'un rôle qu'on leur connaît ou qu'on leur fait jouer. Le rôle est ici indivisible 
de quelque manière, ou sous un angle quelconque, d'une position de désir : désir de jouer le rôle, désir de pervertir le rôle, désir de désinvestir le rôle, de le transgresser, désir de soutenir le rôle pour un sens encore inconnu et à découvrir. Ce qui semble donc vraisemblable c'est que l'ordre du pouvoir social ne puisse s'épargner de passer par l'ordre du signe et que celui-ci ne puisse devenir signifiant sans que ne soit sollicité, à travers la représentation de celui qui parle, le désir de celui qui voit ou écoute. Non point détermination en dernière instance mais motivation sur un fond de détermination psychique organisée (et donc toujours potentiellement désorganisable). L'ordre du pouvoir, sans n'être jamais celui du désir, en est tout aussi inséparable que le désir lui-même l'est de l'organisation socioéconomico-culturelle qui le précède, qui lui propose ses objets, qui le socialise et l'intègre dans un champ symbolique, qui le domestique (dans le double sens de donner un toit et de toiturer le désir) et lui laisse, seion les circonstances, selon le bon vouloir du pouvoir, selon son degré d'émancipation et de libéralité, ou selon l'aptitude critique (ou crisique) du sujet, une marge plus ou moins flexible de manœuvre pour cerner, explorer et découvrir pour luimême et par lui-même, sur fond de pouvoir culturel et de significations sanctionnées, les sentiers et les voies de la signification possible du réel pour l'homme: expérimenter la mouvance qui se situe à la jointure de l'Imaginaire et du Symbolique, là où peuvent régner un léger flottement, une incertitude, un doute (socratique?) à propos de ce qu'il en est du réel, de la réalité des signes et des images, de l'usage des signifiants culturels que l'on croit être siens et générés dans le soi-disant tréfonds de sa subjectivité. Régime du doute, à mi-distance entre les ombres et les opinions de la caverne platonicienne d'une part et ce qui s'avérerait être encore une longue remontée vers le soleil, vers l'Idée, vers ce qui a été une fois contemplé puis oublié, qui sait, peut-être vers l'inconscient lui-même, vers ce qui aurait été désavoué, refoulé dans l'impensable, hors de l'exprimable et qui aurait laissé ouvert béant, comme espace de normalité, d'bumanité, de socialité, le lieu terrestre d'un mixte: celui d'une illusionallusion, d'une ambiguité et d'une infirmité immanente au langage, où l'Imaginaire (prendre le mot pour la chose) et le Symbolique (savoir qu'on ne peut accéder à la chose que par le mot) ne cessent de composer à la manière hésiodique du Jour et de 
la Nuit, impensables hors du circuit de leur mutuel et toujours évanescent empiètement.

La question qui se pose ici, c'est, bien sûr, celle du symbolisme constituant, celle de la représentation incontournable en mots et en images qui me constitue déjà avant même que naisse en mon discours une impression de rêve ou de déjà su, ou encore une impression de vérité, de réalité. Je ne puis prétendre ouvrir la bouche et parler de moi-même, de ma société et de ma culture (ou de la culture) qu'à travers un langage et une masse déjà absorbés et investis de signifiants qui constituent déjà, diront certains, mon identité sémantique et d'autres, mon aliénation dans l'ordre sémiotique. C'est que la culture est ici mon objet de discours (ou le sujet de mon discours) alors même que j'y suis déjà constitué en sujet, puisque c'est en elle que je suis né et que c'est à travers elle que je ne cesse de nommer toute chose.

\section{A) DE LA CULTURE-MÈRE À LA CULTURE-GROUPE: \\ OU DE LA NÉCESSITÉ DU MIROIR ET DES INTENSITÉS FLUCTUANTES DE NOS IMAGES SPÉCULAIRES}

Faudrait-il donc remonter la pente vers la culture-corps maternel à l'origine, apparemment déjà signifiante dans la structuration de mon désir avant même que je ne dispose de mots pour en parler comme ob-jet: lieu donc de mon refoulement originaire avant même, dit-on, que naisse la magie du mot, tenant lieu de l'objet, de l'imago, tenant lieu de l'absence, de l'hallucination psychotique primitive, tenant lieu de l'intolérable: que je puisse exister à l'état séparé au risque d'être perdu, distinct de mon origine, exilé de l'objet sans lequel je ne puis advenir comme sujet. Pour me nommer, pour me construire un moi (bien à moi) il faudrait donc d'abord que j'invente, que je crée l'image du corps déjà autre par lequel je puis être, du sein par lequel je puis déjà halluciner un ob-jet, du regard et du dire de l'autre par lequel je puis m'instaurer à distance de moi-même, dans un miroir de sons, de mots et d'images et qui puissent tenir lieu d'un moi-même par évocation verbale et visuelle, qui puissent m'instaurer dans une représentation de moi-même et qui pourtant, paradoxalement, me sont donnés et rendus en écho par la bouche, le regard et le corps de l'autre. Culture-corps maternel puisque déjà je tisse mon 
image à travers la vision et le discours de l'autre. Culture-corps maternel lui-même produit d'une organisation antérieure de désir, de pouvoir et de signifiants, lui-même acculturé donc et qui, par cercles concentriques élargis, tend à devenir culture père, frère, soeur, culture regard et discours de tout autre où je chemine ainsi dans un monde spéculaire, imaginaire, établi sur le déni de la séparation, sur l'ouverture aux identifications aliénantes, sur l'accès au langage et à la métaphore constituante de l'inconscient, mon désir ne pouvant accéder d'abord à quelque énonciation que par la reprise d'un dire, tenant lieu culturel de mon désir. C'est en cette voie spéculaire, tout à fait anthropologique, que je pourrais me mettre en quête d'un discours dissonant, différent, en quête d'une rupture à réaliser et à reprendre indéfiniment et me tisser au cœur même d'une production de sens qui soit mienne, dans le berceau de mon appartenance et de mes racines culturelles, une identité distincte. Différence au cœur de la répétition.

C'est sur cette prémisse, je crois que repose la possibilité d'envisager la culture comme symbolisme représentatif s' inscrivant dans la représentation spéculaire primitive qui me constitue, et m'intégrant cette fois au corps-groupe comme en un corps maternel plus large (la mère patrie...), comme en une enveloppe protectrice, toute culture, en ce qu'elle tendrait précisément à m'instaurer dans un sens du Même, ayant partie liée avec la dimension primitivement narcissique du désir et à sa réalisation dans les identifications aliénantes mais incontournablement nécessaires à l'image du corps, à l'objet partiel, au regard de l'autre, au discours de l'autre: termes par lesquels pourrait être décrit l'effet de relation duelle, de relation de reconnaissanceméconnaissance caractéristique de l'aliénation idéologique et, de façon plus générale, de l'aliénation initiale du sujet dans la culture, dans l'ordre du langage et des signifiants sociaux. L'idéologie, pour reprendre l'heureuse expression de Ricour ${ }^{31}$, ne serait en réalité qu'une surenchère, qu'un effet de plus-value de crédibilité ou de légitimité s'inscrivant, par redoublement, dans le symbolisme représentatif de ma culture, qu'une insistance visant à accentuer, dans l'initiation au sens, l'effet de miroir auquel, par quelque partie

31. Cf. Paul Ricceur, «La structure symbolique de l'action », in Actes de la $14{ }^{e}$ conférence internationale de sociologie des religions, Strasbourg, 1977, publié avec le concours du CNRS, p. 42 
de moi-même (le désir inconscient, le refoulé), je demeurerais encore lié par-delà le temps du narcissisme primaire et de l'Oedipe, et tout adulte que je prétende être devenu. L'idéologie ne serait donc que par ma reconduction à un désir primitif de me reconnaître en une image omnipotente ou rassurante de moimême par laquelle serait ainsi forclose l'angoisse d'une séparation, d'une différenciation sociale. Entre l'idéologie et la culture, il y aurait donc quelque chose comme l'écart apparemment anodin mais sans doute crucial que l'on peut convenir entre la séduction et le simple fait d'être admis en une présence séduisante. Au cœur de toute culture, une visée anonyme d'unification et de reconnaissance duelle. Au cœur de toute idéologie qui me fait tendre à l'illusion de l'bégémonie (sur la chair, sur l'autre classe, sur le troupeau, sur le mal, sur l'ignorance universelle, sur l'infidèle, sur moi-même et quoi d'autre encore...), une visée séductrice de pouvoir, une visée d'unification par absorption, avalage, pour mieux soumettre et pour mieux régner, pour mieux diviser et ultimement pour mieux pousser à son terme un rapport de force, un rapport nécessairement agonistique ${ }^{32}$. Il faudrait sans doute ici repasser longuement par Freud, par Lacan, mais surtout par Winnicott, Green ou Dolto, pour mieux retracer l'enracinement

32. Il y aurait sans doute lieu ici de distinguer comme le font $R$. Kaës et $A$. Mitscherlich différents types d'idéologies : idéologies fanatiques et idéalistes (par identification au Ca et au Moi Idéal), idéologies d'organisation, de reconstruction et de transformation sociales (par identification à l'idéal du Moi), idéologies réactionnaires (par identification au Surmoi rigide ou à l'idéal du moi chimérique), utopies, etc. Cf. Mitscherlich, Vers la société sans pères, Paris, Gallimard, coll. TEL, 1969, PP. 297-335; KAËs, L'idéologie, études psychanalytiques, Paris, Dunod, coll. Psychismes, 1980 , chapitre 4. Ces deux auteurs proposent des typologies qui, bien que forcément abstraites, font déjà voir la difficulté de distinguer très nettement idéologie et culture, la violence symbolique se jouant à divers degrés d'intensité tant dans les pratiques culturelles que dans les pratiques idéologiques. En ce sens les idéologies pourraient être considérées comme des formes culturelles spécifiques aux groupes engagés dans la poursuite et dans l'exercice du pouvoir politique. La tendance plus ou moins accentuée à la fermeture du sens, à la réification du discours, à l'accentuation de la détermination au dépend de l'indétermination, serait un trait plus particulièrement propre aux idéologies, ne fut-ce que parce que le discours soutient ici une visée d'organisation, de cohésion et d'efficacité stratégique, un effet de frappe, et parce que là où l'intérêt est étroitement concerné et mis en jeu, tout groupe tend à évacuer les interrogations critiques qui risqueraient de morceler ses énergies. En un mot, plus la conjoncture est féroce, plus la pensée tend à régresser vers le fantasme, comme défense contre l'angoisse. Cf. aussi à ce propos l'article de Franco CRESPI, « Médiations symboliques et pouvoir ", in Recherches sociologiques, $\mathrm{n}^{\circ} 1-2$, 1982, pp. 171-180. 
anthropologique du désir dans la culture-représentation et de la culture dans l'ordre du désir : savoir lire où, quand et comment se tisse l'Imaginaire singulier dès les premiers âges de la vie et son emboîtement dans l'Imaginaire socio-culturel, quand ce n'est déjà dans l'Imaginaire socio-politique. Où, quand et comment, pour reprendre l'idée centrale de W. Reich, le désir s'enclenche par une mise en forme caractérielle dans l'ordre de la répression idéologico-politique ou dans l'ordre de ce que Marcuse appelle une surrépression, entendu que l'entrée dans la culture et l'effet de socialité lui-même ne sauraient être sans un état de névrotisation minimal (Freud), sans une répression de premier degré (Marcuse), sans donc un certain refoulement. L'aliénation, la distorsion du sujet dans l'idéologie ne serait point effectivement, pour citer ici Véron ${ }^{33}$, une déformation ou une occultation d'un prétendu réel se produisant sur le terrain épistémologique d'une vérité déjà présente dans les choses elles-mêmes bien que, dira-ton, voilée, mais plutôt le maintien du sujet dans une position désirante narcissique, fétichiste et idéalisante où l'accès à l'ordre symbolique ne peut que s'avérer refusé, retardé ou bloqué. Dominer par les mots, c'est en effet désirer que l'autre n'advienne pas par lui-même à l'énonciation de sa propre parole. Ce n'est pas tant lui mentir à propos du réel, c'est plutôt le circonvenir, le circonscrire dans l'impossibilité de créer ou d'apprendre à créer le sens de sa propre expérience du réel. C'est le gaver de réponses cobérentes et solides à même de capter le désir avant même d'avoir éveillé le sujet au risque de poser ses propres questions. L'idéologie, en ce sens, concerne beaucoup moins une épistémologie du vrai et du faux, du scientifique et du mytbique et bien plus une pédagogie de la libération et de l'inhibition de la parole.

B) DE LA FRAGMENTATION DE L'IMAGE SPÉCULAIRE

À L'ESPACE TRANSITIONNEL: OU DE LA GENĖSE

DU DÉSIR DE S'INVENTER DANS SON

RAPPORT FRAGILE AU DÉSIR DE L'AUTRE

Si toute entrée dans la culture peut donc être considérée dans un premier temps logique comme inscription du sujet dans une représentation spéculaire de ce qu'il est et de ce qu'est le réel par où

33. Cf. E. VERON, op. cit. 
il accède, à travers le regard et le dire de l'autre, à un sens communément partagé de l'ordre du monde, à une structuration première de son propre regard, on peut dans un second temps envisager le processus des identifications spéculaires, comme phase transitionnelle conduisant ultérieurement à une personnalisation possible de l'individu-sujet socialisé, déjà interpellé dans et par la culture, dans et par l'idéologie. Transitionalité qui implique la possibilité d'identifications plus souples, permettant, par-delà la spécularité, l'épreuve de réalité, l'apprentissage de l'altérité (de tout ob-jet), l'apprentissage donc de la dés-illusion, de la précarité et de l'insuffisance des modèles culturels et des signifiants sociaux, et donc l'apprentissage objectivant de l'illusion spéculaire comme partie intégrante et constituante de la réalité sociale. Reconnaître la spécularité comme ciment social ne signifie pas pour autant la reléguer dans le tiroir des illusions perdues, des distorsions et des mystifications infantiles, ni même surtout s'en démettre soimême. Saurait-on en effet s'affranchir de l'inconscient, de la métaphore constituante de l'inconscient, sans qu'il y ait là aussi une perte de plaisir et de désir créateur ? Peut-être cela signifie-t-il plutôt consentir à la perte de ses évidences d'antan, de ses idoles et de ses certitudes antérieures et trop bien établies, ce qui ne va jamais sans affrontement avec les aléas déroutants du doute, fut-il cartésien ou socratique, sans renégociation de l'angoisse comme condition initiale et fondatrice de toute ré-élaboration de la pensée, et donc sans retour forcé aux petites terres de son humanité. Car la question n'est pas ici qu'épistémologique au sens restreint du terme, mais d'abord et avant tout existentielle. L'apprentissage de la tolérance active du paradoxe de dire les choses concerne en effet beaucoup plus l'émergence de la conscience comme expérience progressive et indéfectiblement douloureuse de dénarcissisation du moi (comme intégration donc de l'Imaginaire et du Symbolique) que le simple stockage mental des connaissances éprouvées. Et sans doute cet apprentissage, qui fait étroitement écho à la pédagogie socratique de l'enfantement et de la rupture, présupposerait-il optimalement et, disons-le, idéalement, cela même qu'il se trouve à viser: à savoir cet art d'aimer, dont nous parle E. Fromm, qui n'est que l'autre nom de la liberté et qui, rendant toutes choses possibles, ne fait qu'un en réalité avec la conscience même de l'altérité. En sorte que connaître et aimer ne feraient bien ici idéalement qu'une seule et 
même chose, la double articulation du désir et de la conscience (de soi et de l'autre) étant ce qui consacre dans l'humanité la poursuite et la pertinence de tout autre savoir. Il faut bien reconnaître pourtant que c'est ici justement que le désir achoppe sur une double limite : celle de l'angoisse et celle du pouvoir, du savoir et de l'avoir comme possessions suturantes. Mais peut-être est-ce le fait même de buter sur la limite qui rend aussi probable et nécessaire la quête et le désir du dépassement et du possible.

Ainsi donc conçu, dans un temps second, comme processus transitionnel (débouchant sur une entrée dans une relation d'objet triadique où la représentation se donne non plus comme objet de reconnaissance-méconnaissance mais comme médiation à investir dans l'établissement d'un rapport symbolique au réel) l'Imaginaire nous permet d'envisager les représentations plus ou moins stéréotypées de la culture comme supports et médiations à investir dans un processus de création et d'auto-création conduisant dans des conditions sociales libérantes du désir et de la parole, à la découverte progressive, à travers la créativité au sens large, des multiples versants de la réalité :

a) que ce soit dans les rapports interindividuels (recherche d'authenticité, de vérité, de connaissance des singularités plutôt que reconnaissance en miroir), au sens de recul et de prise de conscience de ce qu'il pourrait y avoir d'illusoire dans toute représentation (jusqu'où l'authenticité est-elle possible à l'encontre de la mauvaise foi? , dirait Sartre, dans toute l'intransigeance... de son vocabulaire).

b) que ce soit dans la prise de conscience des réalités de l'univers physique ou social, comme c'est le cas dans le développement de la connaissance scientifique,

c) que ce soit encore dans le développement du sens esthétique qui, ainsi que le remarque Ernst Kris ${ }^{34}$, présuppose une prise de distance mitoyenne (ni de trop près, ni de trop loin) permettant d'apprécier la justesse et l'à-point de la métaphore, du symbole, de la figure ou de la mise en scène littéraire, picturale, comme expressive, dans la dimension imaginaire, de la condition humaine.

34. Cf. Ernst Kris, Psychanalyse de l'art, Paris, P.U.F., coll. Le fil rouge, 1978. 
Prise de distance qui est à la fois retrait de l'Imaginaire et entrée dans les jeux fascinants de l'Imaginaire où celui-ci se trouve reconnu dans sa fécondité allusive et expressive du réel: connaissance qui se joue au cœur de la reconnaissance.

Si donc l'Imaginaire peut se donner à voir, du point de vue de son efficace sociale, dans sa fonction d'intégration (ou de désintégration) des groupes sociaux et dans sa fonction de motivation collective à l'action et à la résolution de tâches pratiques, il est par contre indéniable que l'Imaginaire est aussi ce par quoi tout sujet, déjà enfant, construit, ou tente de construire, dans la représentation et dans l'énonciation du sens, son rapport à lui-même et à son environnement naturel et social (création d'une identité personnelle et même création incessante d'une identité groupale qui se renouvelle). L'étude des processus de la créativité ou de la création culturelle pose indéniablement des problèmes multiples en ce qu'il s'agit là d'un niveau particulièrement complexe de l'expérience humaine qui résiste largement aux hypothèses explicatives. Néanmoins l'analyse de tels processus (par la psychanalyse ou la sociologie de la création, de l'art, de la littérature) a ceci d'avantageux qu'elle tend à rendre à l'Imaginaire (tant du côté du créateur que du lecteur (au sens large) qui doit recréer pour son propre compte le sens de l'œuvre) sa fonction éminemment dynamique et constituante dans l'accès à une parole pleine, à une réalisation et à une libération personnelle du désir comme signifié, comme introduit donc dans l'ordre de la communication culturelle, dans l'ordre d'une communication possible du sens, là où personne et culture se disent dans leur étroite solidarité dialectique et anthropologique. Relevant de l'illusion, comme réalisation imaginaire du désir, l'œuvre peut être considérée certes comme une échappée dans la résolution provisoire des conflits personnels mais aussi comme une dramatisation ou une symbolisation objectivante de la condition humaine. L'œuvre dit, sous le mode de l'Imaginaire ou en recourant aussi à l'Imaginaire, ce que le commun des mortels n'arrive pas ou n'arrive que difficilement à se représenter, ce qui n'accède que difficilement à l'énonciation et à la parole. Contrairement à l'Imaginaire de l'idéologie, constituant de l'homogénéité plus ou moins étroite des groupes dans la 
représentation, l'Imaginaire créateur se trouve ici rétabli dans sa relation dialectique au Symbolique et au réel. L'écrivain, l'artiste, le sociologue, le politicologue, le philosophe, le poète évoquent dans l'ambiguité esthétique ou dans la production d'un sens nouveau la multidimensionalité du réel (réel affectif, réel comportemental, réel des espaces et des formes, réels des mots, des hommes et des choses), le jeu des singularités et des différences contradictoires, en sorte que chacun puisse s'y reconnaître, s'y interpréter et s'y retrouver. C'est dire que le créateur assume les risques et les obscurités de l'inconscient, le non-dit, l'indicible au coeur de l'homme, l'indicible au cœur du monde lui-même, en leur donnant une forme expressive symbolique qui en assure la maîtrise et la figuration signifiante dans leur passage à la conscience. Ainsi toute culture est plus que la somme fonctionnelle des ensembles signifiants qui régissent la cohésion des groupes et des collectivités. Elle est aussi ce par quoi la représentation spéculaire de groupe se donne à voir dans son insuffisance et dans son impossibilité d'épuiser la production du sens. Et s'il peut arriver que le créateur puisse être l'idéologue confirmé d'un parti, d'un groupe, d'une classe, il est manifeste qu'en tant que créateur il se situe dans la marge du pouvoir social (et non au-delà du pouvoir), dans cet horizon ouvert où la culture est aussi production inépuisable et intarissable du désir singulier, là où l'abstraction réifiante, le culte de l'idée systématique, le clivage manichéen ou la fermeture relative des signifiants socio-culturels cèdent le pas à l'imagerie ou à l'idée suggestives.

L'analyse des processus de la création culturelle nous reconduit donc encore ici à une représentation mobile et mouvante de la culture, là où trop souvent on a tendance à la réduire à des ensembles finis et schématiques, cloués sur eux-mêmes, à une sorte de principe d'identité. Il faudrait voir précisément en quoi toute création, dans sa mobilité même, traduit aussi et ainsi, à sa mesure, les nœuds et les contradictions de la société, ses dilemmes, ses blocages, ses crises, ses ruptures, ses régressions, ses temps de stagnation, de pacification, et parfois ses dépassements. Non pas qu'il s'agisse ici de postuler une sorte de parallélisme, d'effet de résonnance mécanique, entre le vécu du créateur et le devenir des institutions. Mais plutôt penser d'une part que les rythmes de scansion du temps social sont aussi ce qui débusque le désir de ses 
lieux de repos, de ses campements, de ses citadelles, de ses barricades dans la sphère du sens ou, à l'encontre, ce qui peut inciter le créateur aussi bien que tout acteur ou tout groupe social à se cantonner dans la représentation gratifiante d'un rêve, d'une répétition obsédante du même, restauratrice de soi et du monde dans le cercle clos et bien fermé de l'idéologie, de l'idole, de l'idéalité, lorsque le devenir du monde ne saurait être pensé et vécu sans dommage et sans risque de destruction dans l'économie personnelle et groupale du désir. Peut-on penser d'autre part que le constat et que le ressouvenir ou l'anticipation du devenir social, des événements sociaux, en l'un ou plusieurs de leurs modes de scansion (intégration, désintégration, guerre, apogée, luttes etc.), peuvent aussi fournir dans l'ordre du préconscient cette matière perceptive qui peut venir engrosser, féconder l'inconscient du créateur, lui fournir un thème, des objets, des formes à investir pour que soit ainsi libéré le désir et libérés tout autant les fantasmes inconscients, ceux-là autour desquels se trouve organisé, comme en une sorte d'infrastructure ou de grammaire générative de la parole, le désir infini d'une rencontre de l'Objet: le roman historique, le roman de fiction, l'œuvre de l'historien, l'interprétation de l'analyste ou du philosophe, toute œuvre créatrice de sens jaillit bien de cette rencontre dialectique entre l'événement objectif et le désir subjectif, entre la forme perçue et retenue et le désir qui réaménage le regard que l'on porte sur elle et qui, à travers elle, s'essaie à la production d'un sens possible de soi et des choses, de soi à travers les choses, des choses à travers soi. En ce qu'elle est rencontre de l'intérieur et de l'extérieur, et production dans l'extériorité de la forme d'un désir antérieurement non formulé ni formulable, toute œuvre est risque de voir se profiler devant soi un sens nouveau à propos de soi-même et du monde. Et peut-être est-ce précisément parce que nous ne saurions disjoindre ce qui est conjoint, ni rendre conjoint ce qui est disjoint - le corps-sujet désirant et l'objet désiré et donc résistant à l'accomplissement intégral du désir (la pulsion n'étant elle-même jamais totalement satisfaite ni épuisée par l'objet représenté) que la représentation, par ses fluctuations, sa mouvance et ses hésitations à prendre forme et par son impuissance à se matérialiser (à devenir l'objet lui-même) est précisément le seul lieu d'une rencontre possible entre l'un et l'autre, d'une fusion toujours provisoire et à reconduire toujours plus loin et d'une 
séparation aussi intolérable que constituante du dire comme conscience, conscience du conjoint-disjoint, du sujet et de l'objet, entre lesquels s'insèrent comme sutures plastiques l'image, le mot et le symbole.

C'est donc que l'effet de socialisation, de conduction précisément du désir dans l'ordre du général, de l'objectif, du groupe comme entité déjà autre et plus que la simple somme de ses sujets, mais plutôt comme ce qui les relie et les cimente en un être distinct, ayant apparemment une logique propre, une culture (qui est aussi une occult-ure), c'est donc que l'effet de socialisation ne peut lui aussi, tout comme l'effet de création, que passer par la mobilisation des fantasmes inconscients individuels comme formes premières et primitives d'organisation et d'explicitation du désir et par leur retraduction dans la lecture de l'événement, de l'objet, qui serviront de soutien à leur appropriation symbolique, à leur circulation symbolique, à leur structuration au niveau des processus d'élaboration secondaire. Le terme «culture», en ce qu'il suggère l'idée d'un Imaginaire collectif par-delà l'imaginaire singulier, individuel, ne peut donc en fait être que par ce dernier, que par un effet de résonance fantasmatique possible entre les membres d'un groupe ${ }^{35}$, que par une polarisation dirigée, éduquée des fantasmes inconscients individuels sur des représentations d'objets privilégiées, choisies, cultivées, reproduites. Il suffira que celui, celle ou ceux qui ont le pouvoir (ou le devoir) d'évoquer de telles représentations puissent aussi éveiller et polariser en ces représentations les fantasmes individuels (du fait qu'ils sont euxmêmes aimés ou détestés — donc désirés en leur discours) pour que soit rendu possible un imaginaire de groupe, un imaginaire collectif : imaginaire tantôt créateur, tantôt crispé, laissant place à l'ouverture ou à la fermeture selon la logique du désir qu'induit ou que nourrit l'éducateur qui consciemment ou inconsciemment a su se rendre désirable: Hitler, Mussolini, Gandhi, Jésus-Christ, Bouddha... Question de pédagogie ou de moyens pédagogiques sans doute, mais aussi question de besoins et d'urgences conjoncturels inclinant à l'émergence d'un nouveau sens requis par ce qui autrement risquerait d'être vécu sous le coup intolérable

35. Cf. à ce propos l'ouvrage de D. ANZIEu, Le groupe et l'inconscient, Paris, Dunod, coll. Psychismes, 1981, chapitres 4 et 10. 
de l'absurde: illusion compensatoire et mortifère ou fiction constructive et heuristique? Qui saurait prédire le sort de l'Imaginaire social dont l'ambiguité tient à l'ambiguité même du désir au pouvoir? Ne sommes-nous point tous, sous ce rapport, concernés? Ne sommes nous point tous en fait, de quelque manière, éducateurs et éduqués, désirés et désirants, et donc, à ce titre, lieu de circulation d'un pouvoir? Ne sommes-nous point, selon les circonstances, libérateurs ou libérés mais aussi sans doute bourreaux et victimes à l'échelle des micro-pouvoirs et des moyens de pouvoir dont nous disposons dans la circulation du sens?

\section{DÉCADENCE OU ÉLOGE DU POUVOIR}

OU DE L'HOSTILITÉ À LA CONVIVIALITÉ

Que les normes, les valeurs, les idéaux, les interdits, les règles d'une culture, tels qu'ils se trouvent concrètement véhiculés dans les institutions (économiques, politiques, scolaires, familiales, etc.) forment cette toile de fond qui permet aux individus de s'identifier à leur collectivité d'appartenance, que la culture soit ainsi instauratrice d'un ordre relativement stable dans la représentation, que la culture enfin, pour reprendre le discours de Freud, soit cette "Loi symbolique fondée sur les interdits de l'inceste et du meurtre du Père ${ }^{36}$ comme lois premières, prototypes de toute autre loi, permettant par là l'émergence du désir comme délai, comme attente, comme force motrice à investir dans les œuvres de civilisation, tout cela, par ailleurs, ne nous dit pas si la Loi est juste ou arbitraire, si le Père est juste ou arbitraire, si le pouvoir économique est juste ou arbitraire, si le pouvoir politico-institutionnel est lui aussi juste ou arbitraire, si donc la culture est cuvre de libération ou opération de séduction ou de castration du désir. Dira-t-on, en effet, de la culture nordaméricaine, qui est culture de néo-capitalisme, qu'elle est ellemême séduction et par là détournement de la Loi, captation du désir dans une spécularité duelle ${ }^{37}$ et que la culture soviétique est plutôt castration du désir et de la création plutôt qu'instauration du désir et de sa libération dans l'affirmation socio-culturelle des

36. Par Loi j'entends ici tout Ordre de relations sociales comme fondateur du sens

37. Cf. Christopher LaSCH, Le complexe de Narcisse, Paris, Robert Laffont, coll. Libertés $2000,1981$. 
différences? Ou doit-on dire plutôt, en s'épargnant de telles simplifications, qu'en chaque culture se joue un équilibre mal assuré entre la libération et l'aliénation, entre l'imagination créatrice et l'imaginaire crispé de l'idéologie au pouvoir, et que la dynamique culturelle requiert d'être comprise sur la base d'une analyse des conflits, des ruptures, des continuités et des renversements qui ponctuent le trajet plus ou moins vacillant du désir dans son inscription polymorphe, tantôt relevant d'Éros tantôt de Thanatos, dans les jeux de pouvoir, celui-ci se trouvant poursuivi tantôt pour lui-même, en vue de sa pure et simple jouissance (Thanatos), tantôt poursuivi dans une visée libérante, organisatrice et promotrice du plaisir que procure toute création, toute convivialité. D'une part un pouvoir qui ne cesse de se rappeler à lui-même et aux autres, qui ne cesse de reformuler sous des formes variées le fantasme de la maîtrise, de la possession, le fantasme de la puissance absorbant toute participation dans l'illusion phallique ou dans la jouissance symbiotique, d'autre part un pouvoir qui peut s'oublier lui-même comme objet de jouissance et d'identifications aliénantes pour n'être plus que cette référence, référence capitale, qui permet l'émergence des désirs singuliers, des différences singulières, des œuvres singulières dans le plaisir de la reconnaissance mutuelle, de l'échange, de la communication réciproque, de l'enrichissement collectif. Pseudo-libéralité et libéralité du pouvoir. Pouvoir poursuivi comme fétiche ou objet idéal englobant et unitaire, et pouvoir restitué dans sa fonction d'émancipation, de réjouissance, de retrouvaille communautaire. Pouvoir castrant et générant l'obsession et la soif de pouvoir et pouvoir médiateur permettant la reconnaissance de ses frères et de ses sœurs, de la fratrie et de la sororité. Pouvoir qui se joue ainsi, en chaque discours singulier, sur l'un ou l'autre registre ou dans l'oscillation entre l'un et l'autre. Pouvoir qui se joue dans l'État, dans les institutions, dans les groupes, dans la famille, sur le mode de la libre expression du désir ou sur le mode de l'évacuation du désir (qui ne laisse alors qu'un vide à remplir à tout prix). Pouvoir ouvert et pouvoir pervers, pouvoir d'émulation et pouvoir de dominance, pouvoir qui fait croître et grandir et pouvoir qui donne l'illusion d'être sans manque et par conséquent de tout temps omnipotent, sans histoire et sans devenir. Pouvoir qui rend possible l'assomption de l'incertitude, du risque, de l'invention, de la temporalité et de ses fluctuations et pouvoir qui veut se fixer 
dans l'absolu d'un moment éternel. Absolu d'un éternel présent qui est en tout point à l'inverse de l'absolu du moment de la création, pressenti intensément comme mouvance à dire, à domestiquer dans l'ordre des signes et du langage, dans l'ordre de la culture.

Il y a sans doute lieu de conclure ici que la culture peut nous apparaître tout compte fait elle aussi comme un espace potentiel et intermédiaire entre le groupe social comme réalité référentielle, comme matrice originelle et vivante du sens du réel, et le corps propre de tout sujet désirant comme explorateur et porteur d'une expérience irréductible de l'espace naturel et social. La culture serait donc cet espace intermédiaire de symbolisation où les pratiques sociales peuvent s'avérer prendre $d u$ sens ou, au contraire, donner cours au sentiment d'une perte de sens selon que le désir peut se chercher ou doit se nier, pour se trouver ou pour se démettre, dans le cadre des filières et des significations socialement élaborées et institutionnellement gérées en vue de la satisfaction des besoins humains. Toute société s'appuyant ainsi solidairement sur le désir aussi bien que sur le pouvoir, ce que jappelle le non-sens pourrait dès lors se dire non seulement de l'impuissance advenue de satisfaire matériellement les besoins humains les plus élémentaires, ce que toute société totalitaire ou énergumène peut encore assez aisément réaliser, mais aussi de l'Impuissance à porter au niveau manifeste du sens le comment et le pourquoi des conflits, des misères, des tensions, des malaises aussi bien que des joies et des satisfactions de la vie quotidienne d'un groupe ou d'une nation tout entière. Toute répression sociale implique toujours sous ce rapport une dépossession de la parole comme cuvre de désir et de création du sens aussi bien qu'une privation de l'accès aux moyens d'expression et d'expérience culturels qui en rendraient l'élaboration possible: ce que Paulo Freire a su fort bien mettre en évidence dans ses considérations sur la pédagogie des opprimés ${ }^{38}$. Car ce n'est pas tant de signifiés que manque le discours muet de l'opprimé mais bien plutôt de signifiants capables d'éveiller une parole confisquée. Mais les opprimés, faut-il le faire remarquer, ne sont pas toujours, dans cette perspective désirante, les seuls sujets économiquement

38. Paulo Freire, Pédagogie des opprimés, Maspero, PCM, 1980. 
démunis. Car il y a bien aussi, forcément, une aliénation du désir néo-bourgeois qui, pour arriver à l'étroitesse de sa jouissance, doit bien aussi apprendre à se taire et à rentrer dans les rangs. À moins qu'on ne lui demande en exédent de donner l'exemple en échange d'une parcelle supplémentaire de pouvoir, de revenus et de privilèges. Aussi est-ce bien du silence et des bruits informes et inarticulés que s'alimente l'opacité du discours idéologique, du discours de légitimation, pour autant que l'idéologie devient le lieu par excellence de signifiance (ou d'insignifiance) d'où ne peut émerger aucune parole. Le non-sens serait bien en conséquence l'effet de la fragmentation d'un espace potentiel culturel de moins en moins plastique, de plus en plus inconsistant, de moins en moins articulable, ou de plus en plus rigide, ouvrant sur la dissolution de toute communication vivante et sur un clivage marqué entre les représentations culturelles comme donatrices originelles de sens et les pratiques sociales effectives des hommes comme véhiculant, au cœur même du discours culturel sanctionné et promulgué, un contre-sens inavoué et inavouable: sociétés culturellement schizophréniques, sociétés de la double contrainte (double bind) ou, à l'encontre, sociétés culturellement paranoïaques, mais dans tous les cas sociétés où le cadre culturel du sens, qui devrait ici servir de toile de fond sur laquelle puisse s'effectuer, en s'en démarquant, tout processus de création et d'inscription mobilisateur des énergies actives du désir, serait lui-même devenu une coquille vide.

Que les cultures s'évanouissent et disparaissent dans la dissolution lente et parfois même dans la violence la plus précipitée et la plus insensée, voilà bien ce que nous enseigne l'histoire d'hier aussi bien que celle des temps présents. La cécité des pouvoirs se trouve alors à générer elle-même sa propre subversion, que ce soit par une excroissance pathologique de l'imaginaire idéologique dans le déni ou le désaveu des problèmes réels que pose la coexistence collective et la coexistence entre les peuples ou que ce soit dans les flambées de violence qui se trouvent à disqualifier sans retour possible la plausibilité ou la crédibilité d'un système de représentations et de valeurs collectives. Qu'en retour, toute dissolution culturelle en appelle à une renaissance, à la production d'un nouveau pouvoir, illustre bien que toute émancipation du désir créateur ne peut passer que par une loi 
sociale, que par une nouvelle matrice d'identité, dans la reconnaissance mutuelle des différences, des œuvres singulières, instauratrice donc du respect des différences, instauratrice de cadres viables d'existence ouvrant sur un sentiment de solidarité et de réciprocité. Toute création, en ce sens, est rappel du caractère désuet du pouvoir (politique ou politico-culturel) ou de son insuffisance, ou encore appel à un nouveau dépassement dans l'ordre de penser les hommes et les choses. Toute création est appel à rendre au pouvoir sa mobilité, à ne point s'y fixer, à ne point le fétichiser. Aussi ne faut-il point s'étonner que le pouvoir puisse parfois se raidir devant la création, quand il n'en fait pas son commerce, ou encore qu'il puisse se louer et jouir dans le plaisir d'avoir mis au monde ses créateurs.

Département de philosophie, Université du Québec à Trois-Rivières. 